\title{
Candida albicans-Induced NETosis Is Independent of Peptidylarginine Deiminase 4
}

\author{
Eva Guiducci ${ }^{1}$, Christina Lemberg ${ }^{1}$, Noëmi Küng ${ }^{1}$, Elisabeth Schraner ${ }^{2}$, \\ Alexandre P. A. Theocharides ${ }^{3}$ and Salomé LeibundGut-Landmann ${ }^{1 *}$ \\ 'Section of Immunology, Vetsuisse Faculty, University of Zurich, Zurich, Switzerland, ${ }^{2}$ Institute of Virology, Vetsuisse Faculty, \\ University of Zurich, Zurich, Switzerland, ${ }^{3}$ Division of Hematology, University Hospital Zurich, University of Zurich, Zurich, \\ Switzerland
}

\section{OPEN ACCESS}

Edited by:

Martin Herrmann, Universitätsklinikum Erlangen, Germany

Reviewed by: Luuk Hilbrands,

Radboud University Nijmegen, Netherlands Yi Zhao,

West China Hospital of Sichuan University, China

*Correspondence: Salomé LeibundGut-Landmann salome.leibundgut-landmann@uzh.ch

Specialty section:

This article was submitted to Molecular Innate Immunity, a section of the journal Frontiers in Immunology

Received: 03 April 2018 Accepted: 25 June 2018

Published: 09 July 2018

Citation: Guiducci E, Lemberg C, Küng N, Schraner E, Theocharides APA and LeibundGut-Landmann S (2018) Candida albicans-Induced NETosis Is Independent of Peptidylarginine Deiminase 4.

Front. Immunol. 9:1573. doi: 10.3389/fimmu.2018.01573
Neutrophils are the most abundant innate immune cells and the first line of defense against many pathogenic microbes, including the human fungal pathogen Candida albicans. Among the neutrophils' arsenal of effector functions, neutrophil extracellular traps (NETs) are thought to be of particular importance for trapping and killing the large fungal filaments by means of their web-like structures that consist of chromatin fibers decorated with proteolytic enzymes and host defense proteins. Peptidylarginine deiminase 4 (PAD4)-mediated citrullination of histones in activated neutrophils correlates with chromatin decondensation and extrusion and is widely accepted to act as an integral process of NET induction (NETosis). However, the requirement of PAD4-mediated histone citrullination for NET release during C. albicans infection remains unclear. In this study, we show that although PAD4-dependent neutrophil histone citrullination is readily induced by $\mathrm{C}$. albicans, PAD4 is dispensable for NETosis in response to the fungus and other common NET-inducing stimuli. Moreover, PAD4 is not required for antifungal immunity during mucosal and systemic $C$. albicans infection. Our results demonstrate that PAD4 is dispensable for $C$. albicans-induced NETosis, and they highlight the limitations of using histone citrullination as a marker for NETs and PAD4 ${ }^{-/-}$mice as a model of NET-deficiency.

Keywords: neutrophil extracellular traps, peptidylarginine deiminase 4, Candida albicans, host-fungus interaction

\section{INTRODUCTION}

Candida albicans is one of the most common etiological agents of opportunistic fungal infections that occur predominantly in immunocompromised individuals but can also affect healthy individuals, e.g. as a consequence of antibiotic therapy. C. albicans-mediated diseases range from mild superficial infections affecting the oral and vaginal mucosa to life-threatening bloodstream infections (1). Invasive candidiasis remains most difficult to diagnose and to treat and is thus associated with a high mortality rate. The development of clinical symptoms depends on both the host immune status and microbiota and on C. albicans virulence traits. Among those, C. albicans has the ability to reversibly switch between spherical yeast cells and large filaments called hyphae (2). While the yeast form is thought to be relevant for dissemination, hyphal growth is essential for tissue invasion and destruction (3). C. albicans filamentation is associated with increased expression of virulence factors, including adhesins, invasins, and hydrolytic enzymes that contribute to disrupting epithelial and endothelial barriers $(4,5)$. A robust immune response is required to protect the host against C. albicans infections $(4,6)$. Neutrophils are polymorphonuclear leukocytes of the innate immune 
system that constitute the first line of defense against C. albicans infections limiting fungal growth and dissemination (7). The high incidence of candidiasis in neutropenic patients as well as data from experimental studies in mice provide evidence for the critical protective role of neutrophils in antifungal defense $(8,9)$. Neutrophils can, however, also contribute to immunopathology and organ failure in some conditions $(10,11)$. Neutrophils are rapidly recruited from the bloodstream to the site of infection, where they engulf and kill pathogens by phagocytosis or via extracellular defense mechanisms $(7,12,13)$. While $C$. albicans yeast cells are rapidly phagocytosed, hyphae are too large to be engulfed by neutrophils (14).

The release of neutrophil extracellular traps (NETs) has emerged as a prevalent strategy of neutrophils to combat large microbes (15). NETs are, as their name implies, web-like structures of chromosomal DNA decorated with histones and antimicrobial effector molecules (16), including granular proteins such as neutrophil elastase (NE), myeloperoxidase (MPO), and cytoplasmic proteins such as calprotectin (13). NETs have been widely shown to be induced by and to trap bacterial (12) and fungal pathogens (17) as well as protozoan parasites (18-20). However, NET formation or impaired NET degradation have also been shown to play a pathological role in non-infectious conditions, including the development of inflammatory and autoimmune diseases like systemic lupus erythematosus (21), thrombosis (22), autoimmune vasculitis (23), and psoriasis (24). Moreover, NETs were shown to be involved in metastatic processes, by trapping migrating tumor cells in the microvasculature and promoting the formation of micrometastasis (25).

Neutrophil extracellular trap release requires the production of reactive oxygen species (ROS) by NADPH oxidase (26). ROS promotes the translocation of NE from the azurophilic granules to the nucleus where it digests nucleosomal histones and promotes chromatin relaxation. Subsequently, MPO associates with chromatin and, synergistically with NE, promotes massive chromatin decondensation $(27,28)$. In association with diverse granular and cytoplasmic proteins (13), decondensed chromatin is eventually released into the extracellular space (28).

NETosis was further proposed to involve histone deimination by peptidylarginine deiminase 4 (PAD4) (29, 30). PAD4 is primarily expressed in the nucleus of neutrophils, where it catalyses the conversion of histone arginine to citrulline residues in a calcium-dependent manner (31). Citrullination is a posttranslational modification that plays an important role in many physiological processes, including skin keratinization, brain plasticity, gene regulation, and immune functions. Abnormal citrullination, though, can lead to the development of autoimmune diseases and cancer (32). Numerous stimuli have been reported to lead to PAD4 activation and NET formation (33); these include phorbol 12-myristate 13-acetate (PMA) (33), the calcium ionophore ionomycin (34), and various bacteria, viruses, and parasites (35).

Although PAD4-dependent histone citrullination is widely accepted as a key component in NETosis, firm evidence is still missing. Here, we use PAD4 ${ }^{-/-}$mice to assess the relevance of PAD4 in NETosis and in host protection against C. albicans infection.

\section{MATERIALS AND METHODS}

\section{Mice}

$\mathrm{PAD}^{-/-}$mice on the C57BL/6 background (35) were obtained from late Kerri A. Mowen (La Jolla, CA, USA) and bred at the Laboratory Animal Service Center (University of Zürich, Switzerland). Wild-type (WT) C57BL/6J mice were purchased from Janvier Elevage. In some experiments, $\mathrm{PAD} 4^{+/+}$mice were used as WT, which were obtained by intercrossing PAD4 $4^{+/-}$mice. All mice were kept in specific pathogen-free conditions and used in sex- and age-matched groups at 6-12 weeks of age.

\section{Fungal Strain}

The C. albicans strain SC5314 (36) was used for all experiments except where stated otherwise. The yeast-locked strain $h g c 1 \Delta / \Delta$ and its revertant $h g c 1 \Delta / \Delta: H G C 1$ (37) were obtained from N. Gow (Aberdeen, UK). The oral isolate 101 (38) and strain pACT1GFP (39) were described previously. All strains were grown in YPD medium at $30^{\circ} \mathrm{C}$ and $180 \mathrm{rpm}$ for $15-18 \mathrm{~h}$. For induction of hyphae, $5 \times 10^{3} \mathrm{cfu} C$. albicans yeast cells were incubated in Hanks' Balanced Salt Solution (HBSS; Life Technologies) containing $\mathrm{CaCl}_{2}$ and $\mathrm{MgCl}_{2}$ and supplemented with 5\% FCS for $3 \mathrm{~h}$ at $37^{\circ} \mathrm{C}$ in a humidified atmosphere containing $5 \% \mathrm{CO}_{2}$. In some experiments, C. albicans hyphae were opsonized for $30 \mathrm{~min}$ on ice in HBSS supplemented with $10 \%$ fresh mouse serum (for assays with murine neutrophils).

\section{Murine Infection Models}

For systemic infection, mice were infected via the lateral tail vein with $1.5 \times 10^{5} \mathrm{C}$. albicans yeast cells unless stated otherwise. For oropharyngeal candidiasis (OPC), mice were infected sublingually with $2.5 \times 10^{6} \mathrm{C}$. albicans yeast cells as described (40), without immunosuppression. Mice were monitored for morbidity throughout the course of all experiments. For determination of the fungal burden, the kidneys (systemic candidiasis) or the tongue (OPC) of euthanized animals were removed, homogenized in sterile $0.05 \% \mathrm{NP} 40$ in $\mathrm{H}_{2} \mathrm{O}$ for 3 min at $25 \mathrm{~Hz}$ using a Tissue Lyzer (Qiagen), and serial dilutions were plated on YPD agar containing $100 \mu \mathrm{g} / \mathrm{ml}$ ampicillin.

\section{Preparation of Tongue and Kidney Cells for Flow Cytometry Analysis}

Mice were anesthetized with a sublethal dose of Ketamine $(100 \mathrm{mg} / \mathrm{kg})$, Xylazin $(20 \mathrm{mg} / \mathrm{kg})$, and Acepromazin $(2.9 \mathrm{mg} / \mathrm{kg})$ and perfused by injection of PBS into the right heart ventricle prior to removing the kidneys or the tongue. Kidneys were cut into fine pieces and digested with DNase I (200 $\mu \mathrm{g} / \mathrm{ml}$, Roche) and Collagenase I $(240 \mathrm{mg} / \mathrm{ml}$, Invitrogen $)$ in RPMI at $37^{\circ} \mathrm{C}$ for $20 \mathrm{~min}$. Tongues were cut into fine pieces and digested with DNase I $(200 \mu \mathrm{g} / \mathrm{ml}$, Roche $)$ and Collagenase IV ( $4.8 \mathrm{mg} / \mathrm{ml}$, Invitrogen $)$ in $\mathrm{PBS}$ at $37^{\circ} \mathrm{C}$ for $45-60 \mathrm{~min}$. Single cell suspensions were passed through a $70 \mu \mathrm{m}$ strainer using ice-cold PBS supplemented with $1 \%$ FCS and 2 mM EDTA and analyzed by flow cytometry.

\section{Flow Cytometry}

The single cell suspensions of kidneys and tongues were stained in ice-cold PBS supplemented with 1\% FCS, 5 mM EDTA, and 
$0.02 \% \mathrm{NaN}_{3}$ with LIVE/DEAD Fixable Near-IR Stain (Life Technologies). For staining of surface markers, cells were incubated with fluorochrome-conjugated antibodies against mouse CD45.2 (clone 104), CD11b (clone M1/70), Ly6G (clone 1A8); Ly6C (clone AL-21). All antibodies were from BioLegend. Stained cells were analyzed on a FACS LSRII (BD Biosciences) or on a FACS Gallios (Beckman Coulter), and the data were analyzed with FlowJo software (Tristar). Only live single cells were included in the analysis, and cell numbers were determined using fluorescent counting beads (BD Biosciences; Beckman Coulter).

\section{Histology and Immunohistochemistry of Mouse Tissue Sections}

Mice were euthanized on the indicated day after infection and the kidneys or the tongue were removed, fixed in 4\% PBS-buffered paraformaldehyde overnight, and embedded in paraffin. For histology, tissue sections $(3-5 \mu \mathrm{m})$ were mounted on glass slides and were stained with Periodic-Acid Schiff (PAS) reagent and counterstained with hematoxylin. For immunohistochemical staining, a Dako Autostainer (Dako Autostainer Universal Staining System Model LV-1, Dako-Agilent Technologies) or the Discovery XT platform (Ventana), were used. Tissue sections on poly-L-lysine-coated glass slides were deparaffinized in xylene and rehydrated in a graded series of alcohol. Antigens were retrieved by incubation in citrate buffer, $\mathrm{pH} 6$ at $98^{\circ} \mathrm{C}$ for $20 \mathrm{~min}$. Sections were then incubated with rat anti-mouse Ly6G (clone 1A8, $5 \mu \mathrm{g} / \mathrm{ml}$, BioLegend) or rabbit polyclonal anti-human citrullinated histone $\mathrm{H} 3$ (CitH3, $1 \mu \mathrm{g} / \mathrm{ml}, \mathrm{Abcam}$ ) for $60 \mathrm{~min}$ at room temperature (for anti-Ly6G) or at $37^{\circ} \mathrm{C}$ (for anti-CitH3). After blocking of endogenous peroxidases (Peroxidase blocking reagent; Dako) for $10 \mathrm{~min}$ at room temperature, slides were incubated for $30 \mathrm{~min}$ at room temperature with a detection antibody (biotinylated rabbit anti-rat IgG, $15 \mu \mathrm{g} / \mathrm{ml}$, Vector, followed by avidin-horseradish peroxidase, Vector, for anti-Ly6G; or OmniMap anti-rabbit horseradish peroxidase, Ventana, for anti-CitH3), followed by incubation with 3,3-diaminobenzidine as chromogen and light counterstain with hematoxylin. Images were acquired with a digital slide scanner (NanoZoomer-XR C12000; Hamamatsu) and analyzed with the NDP.view2 software (Hamamatsu). The renal cortex and the tongue epithelium were defined based on anatomical criteria.

\section{RNA Isolation and Quantitative RT-PCR From Mouse Tissues}

Isolation of total RNA from kidney tissues was carried out according to standard protocols using Trizol Reagent (Sigma). cDNA was generated by RevertAid reverse transcriptase (Thermo Scientific). Quantitative PCR was performed using SYBR Green (Roche) and a QuantStudio 7 Flex instrument (LifeTechnologies). The primers were Kim-1 fwd 5-ATGAATCAGATTCAAGTCTTC-3' and Kim-1 rev 5-TCTGGTTTGTGAGTCCATGTG-3; Actb fwd 5-CCCTGAAGTACCCCATTGAAC-3' and Actb rev 5- CTT TTCACGGTTGGCCTTAG-3. All qPCR assays were performed in duplicates and the relative gene expression (rel. expr.) was determined after normalization to $\beta$-actin transcript levels.

\section{Serological Determination of Renal Function}

Blood was collected from infected mice by cardiac puncture and serum was obtained by centrifugation of the clotted blood for $90 \mathrm{~s}$ at $15,000 \mathrm{~g}$ and stored at $-20^{\circ} \mathrm{C}$ until analysis. Blood urea nitrogen (BUN) and creatinine concentrations were determined using QuantiChrom ${ }^{\mathrm{TM}}$ Urea and QuantiChrom ${ }^{\mathrm{TM}}$ Creatinine assay kits (BioAssay Systems) according to the manufacturer's instructions.

\section{Human Peripheral Blood Neutrophils}

Peripheral blood was freshly drawn into EDTA-monovettes by venous puncture from two healthy adult volunteers and two patients with acquired MPO-deficiency. Samples were kept at room temperature during the entire isolation procedure. Neutrophils were freshly isolated over polymorphprep solution (Axis-Shield) by density centrifugation for $35 \mathrm{~min}$ at $450 \mathrm{~g}$, without brake. The polymorphonuclear cell fraction was collected in a new tube and normal osmolarity was restored by adding an equal volume of $0.45 \% \mathrm{NaCl}$. To lyse contaminating erythrocytes, the cell pellet was resuspended in $3 \mathrm{ml}$ of $0.2 \%$ $\mathrm{NaCl}$ for $30 \mathrm{~s}$ followed by addition of $3 \mathrm{ml}$ of $1.6 \% \mathrm{NaCl}$ and $4 \mathrm{ml}$ PBS. The lysis was repeated 1-2 times until no erythrocytes were visible. Neutrophils were then resuspended in HBSS containing $\mathrm{CaCl}_{2}$ and $\mathrm{MgCl}_{2}$ and counted with trypan blue.

\section{Murine Bone Marrow Neutrophils}

Mice were anesthetized with a sublethal dose of Ketamine (100 mg/kg), Xylazin (20 mg/kg), and Acepromazin $(2.9 \mathrm{mg} / \mathrm{kg})$ and perfused by injection of PBS into the right heart ventricle prior to remove the bones. The bone marrow was flushed from femurs and tibiae and passed through a $70 \mu \mathrm{m}$ strainer using ice-cold PBS supplemented with $1 \%$ FCS and $2 \mathrm{mM}$ EDTA. Neutrophils were isolated over a Histopaque-1077 and Histopaque-1119 gradient by density centrifugation at $710 \mathrm{~g}$ for $30 \mathrm{~min}$, without brake. The polymorphonuclear cell fraction was collected in HBSS containing $\mathrm{CaCl}_{2}$ and $\mathrm{MgCl}_{2}$. Erythrocytes were lysed using erythrocytes lysis buffer $\left(0.3 \mathrm{M} \mathrm{NH}_{4} \mathrm{Cl}, 28 \mu \mathrm{M}\right.$ $\mathrm{NaHCO}_{3}, 125 \mu \mathrm{M}$ EDTA). Neutrophil purity was assessed by flow cytometry by staining for $\mathrm{CD}_{4} 5^{+} \mathrm{CD} 11 \mathrm{~b}^{+} \mathrm{Ly}_{6} \mathrm{G}^{+}$neutrophils and was found to be generally between 70 and $80 \%$. The cell numbers used in each experiment were adjusted accordingly to guarantee that equal numbers of neutrophils were present in all experimental groups (e.g., WT vs. PAD4 ${ }^{-/-}$neutrophils).

\section{Quantification of NET Release From Activated Neutrophils}

Freshly isolated human and mouse neutrophils $\left(10^{5}\right.$ cells $)$ were cultured in 96-well tissue culture plates in HBSS containing $\mathrm{CaCl}_{2}$ and $\mathrm{MgCl}_{2}$ and stimulated with PMA (100 ng/ml, SigmaAldrich), Ionomycin $\left(1 \mu \mathrm{M}\right.$, Sigma-Aldrich), or with $5 \times 10^{3}$ C. albicans yeast or preformed hyphae for $2.5 \mathrm{~h}$ at $37^{\circ} \mathrm{C}$. Human neutrophils were treated with the $\mathrm{PAD}$ inhibitor $\mathrm{Cl}$-amidine ( $200 \mu \mathrm{M}$, Merck Millipore) for $2.5 \mathrm{~h}$. The activity of $\mathrm{Cl}$-amidine was confirmed by means of $\mathrm{CitH} 3$ staining. After the incubation, Sytox Green (160 nM, Invitrogen) was added to the cells for 
detection of extracellular DNA. Unstimulated neutrophils were used as controls. The plates were analyzed on an Infinite 200 plate reader (Tecan) with excitation at $485 \mathrm{~nm}$ and emission at $535 \mathrm{~nm}$. The fluorescence of stimulated cells was calculated by subtracting the baseline fluorescence of unstimulated cells and is expressed in arbitrary units. In some experiments, Sytox Green staining was also analyzed by fluorescence microscopy.

\section{Immunofluorescence Staining}

$10^{5}$ isolated human or mouse neutrophils were seeded on glass coverslips in tissue-culture plates and incubated with PMA (100 ng/ml, Sigma-Aldrich), Ionomycin ( $1 \mu \mathrm{M}$, Sigma-Aldrich), or $5 \times 10^{3} \mathrm{C}$. albicans preformed hyphae for $2.5 \mathrm{~h}$ at $37^{\circ} \mathrm{C}$. Cells were then fixed with $4 \%$ paraformaldehyde, permeabilized with $0.01 \%$ TritonX-100 for $10 \mathrm{~min}$, and blocked with 5\% donkey serum for $30 \mathrm{~min}$ at room temperature. Cells were stained with goat polyclonal anti-MPO (R\&D systems), rabbit polyclonal anti$\mathrm{CitH} 3$ (Abcam), and combined rat anti-S100A8 and anti-S100A9 (clone 10 and clone 46 respectively, kindly provided by C. Urban, Umeå, Sweden). Secondary antibodies were donkey anti-goat IgG (Jackson ImmunoResearch), goat anti-rat IgG (Abcam), and goat anti-rabbit IgG (Jackson ImmunoResearch). The PKH26 Red Fluorescent Cell Linker Kit (Sigma-Aldrich) was used to stain lipids of the neutrophil plasma membranes. DNA was stained with $4^{\prime}, 6^{\prime}$-Diamidino-2-phenylindole dihydrochloride (DAPI, Sigma-Aldrich) and NETs were visualized using a Leica SP8 inverse confocal microscope and analyzed with FIJI software.

\section{Scanning Electron Microscopy}

Freshly purified murine bone marrow neutrophils were stimulated on glass objective slides in a 24-well cell culture plate with preformed $C$. albicans hyphae, $100 \mathrm{ng} / \mathrm{ml} \mathrm{PMA}$, and $1 \mu \mathrm{M}$ ionomycin in $\mathrm{HBSS}$ for $2.5 \mathrm{~h}$ at $37^{\circ} \mathrm{C}$ and $5 \% \mathrm{CO}_{2}$, then, cells were fixed with $0.1 \mathrm{M}$ Cacodylate buffer (Merck) containing 2.5\% glutaraldehyde. After washing three times with PBS, the samples were treated with $1 \%$ Osmiumtetroxide in PBS for $30 \mathrm{~min}$, washed again three times with PBS, dehydrated by incubation in $70 \% \mathrm{EtOH}$ in $\mathrm{H}_{2} \mathrm{O}$ for 30 min followed by $100 \% \mathrm{EtOH}$ for $30 \mathrm{~min}$ and an incubation with hexamethyldisilazane (Sigma) for $2 \mathrm{~h}$ prior to air drying overnight. The specimen surfaces were sputter coated with $4 \mathrm{~nm}$ gold/palladium (CCU-010 HV, Safematic, Switzerland) and imaged using a Zeiss Supra 50 VP scanning electron microscope using the secondary electron detector (Zeiss, Oberkochen, Germany).

\section{Transmission Electron Microscopy}

$4 \times 10^{6}$ bone marrow neutrophils were incubated with $5 \times 10^{5} \mathrm{cfu}$ C. albicans preformed hyphae for $2.5 \mathrm{~h}$ at $37^{\circ} \mathrm{C}$. Cell suspensions were centrifuged for $5 \mathrm{~min}$ at $5.9 \mathrm{~g}$, and the supernatants were discarded. Pellets were re-suspended with $2.5 \%$ glutaraldehyde in $0.1 \mathrm{M} \mathrm{Na} / \mathrm{K}$-phosphate, transferred to a micro tube, and centrifuged for $20 \mathrm{~min}$ at $3,400 \mathrm{~g}$. The pellets were rinsed with $0.1 \mathrm{M} \mathrm{Na} / \mathrm{K}$-phosphate and postfixed with $1 \%$ osmium tetroxide in $0.1 \mathrm{M} \mathrm{Na} / \mathrm{K}$-phosphate for $1 \mathrm{~h}$, dehydrated in a series of ethanol starting at $70 \%$, and after transferring into acetone embedded in epon followed by polymerization at $60^{\circ} \mathrm{C}$ for 2.5 days. Sections of $60-80 \mathrm{~nm}$ thickness were stained with uranyl acetate and lead citrate and analyzed in a transmission electron microscope (CM12, FEI, Eindhoven, The Netherland) equipped with CCD cameras (Ultrascan 1000 and Orius SC1000, Gatan, Pleasanton, CA, USA) at an acceleration voltage of $100 \mathrm{kV}$.

\section{C. albicans Killing by Neutrophils}

$5 \times 10^{4}$ neutrophils were added to each well of a 96-well microplate containing $5 \times 10^{3} \mathrm{C}$. albicans preformed hyphae and incubated for $3 \mathrm{~h}$ at $37^{\circ} \mathrm{C}$. The neutrophils were then lysed with water supplemented with $0.02 \%$ Triton-X-100, and the metabolic activity of C. albicans was assessed with Alamar blue (Invitrogen) according to the manufacturer's instructions. A standard curve was generated with serial dilutions of hyphal cells (without neutrophils). Killing activity is expressed as percent of viable fungi in the presence of neutrophils compared to the conditions without neutrophils.

\section{C. albicans Uptake by Neutrophils}

$2.5 \times 10^{4}$ murine bone marrow neutrophils were incubated for $1 \mathrm{~h}$ at $37^{\circ} \mathrm{C}$ with either opsonized or non-opsonized pACT1-GFP C. albicans yeast cells at a fungus:neutrophil ratio of 1:1. After washing, neutrophils were incubated with anti-Ly6G antibody (BioLegend) for $20 \mathrm{~min}$ at $4^{\circ} \mathrm{C}$. The degree of C. albicans uptake was determined by counting the number of $\mathrm{GFP}^{+}$neutrophils relative to the total number of neutrophils by flow cytometry.

\section{ROS Assay}

$2 \times 10^{5}$ mouse neutrophils were added to each well of a 96-well microtiterplate containing $10^{5} \mathrm{C}$. albicans yeast cells or preformed hyphae. Total ROS production was measured by adding cellpermeable luminol (100 $\mu \mathrm{M}$, Sigma-Aldrich), while extracellular ROS was assessed by adding cell-impermeable lucigenin $(400 \mu \mathrm{M}$, Sigma-Aldrich). Chemiluminescence was measured on an Infinite 200 plate reader (Tecan) every $2.5 \mathrm{~min}$ over a total period of $2.5 \mathrm{~h}$ starting immediately after addition of the substrates.

\section{Statistics}

Statistical significance was determined by unpaired $t$ test with Welch's correction or two-way ANOVA using GraphPad Prism (GraphPad Software) with ${ }^{\star} p<0.05 ;{ }^{* *} p<0.01 ;{ }^{* *} p<0.001$. Data displayed on a logarithmic scale were log-transformed before statistical analysis.

\section{RESULTS}

\section{C. albicans-Induced Citrullination of Histone H3 Is Strictly Dependent on PAD4}

We set out to assess the activity of PAD4 in response to C. albicans. Citrullination of histone $\mathrm{H} 3$ (CitH3) was strongly induced in murine bone marrow neutrophils when stimulated with C. albicans hyphae or with ionomycin as a control (Figures 1A,B). Notably, only a fraction of neutrophils acquired the modification with both stimuli. As expected, citrullination was completely absent in neutrophils lacking PAD4 due to a genetic deletion (Figures 1A,B). 
Citrullination of histone $\mathrm{H} 3$ in response to $C$. albicans was also observed in vivo in the kidney of mice that were infected via the intravenous route with strain SC5314 (Figure 1C). The kidney is the primary target organ of the fungus in this model of infection, where C. albicans hyphae spread throughout the renal cortex and eventually extend to the renal tubules and pelvis (41). Neutrophils, and to some extent also inflammatory monocytes, infiltrate the infected kidney and accumulate around C. albicans hyphae forming dense inflammatory abscesses. Induction of $\mathrm{CitH} 3$ in the renal cortex of WT C57BL/ 6 mice occurred as early as $24 \mathrm{~h}$ postinfection and further increased over time (Figure S1A in Supplementary Material). Notably, only a fraction of all Ly6G-positive neutrophils stained positive for CitH3 (Figure S1B in Supplementary
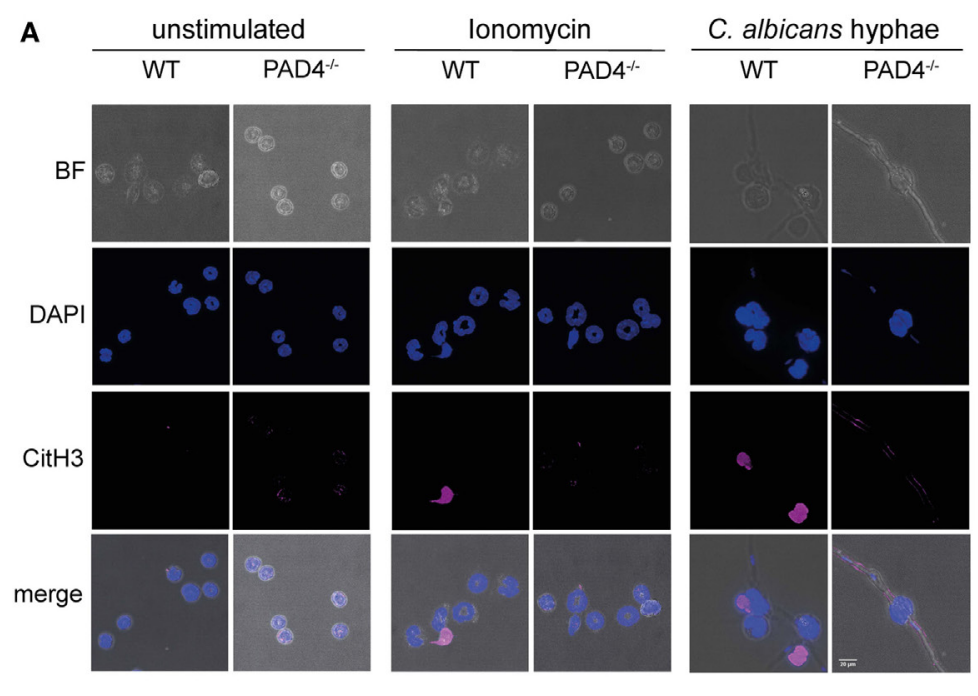

\section{B}
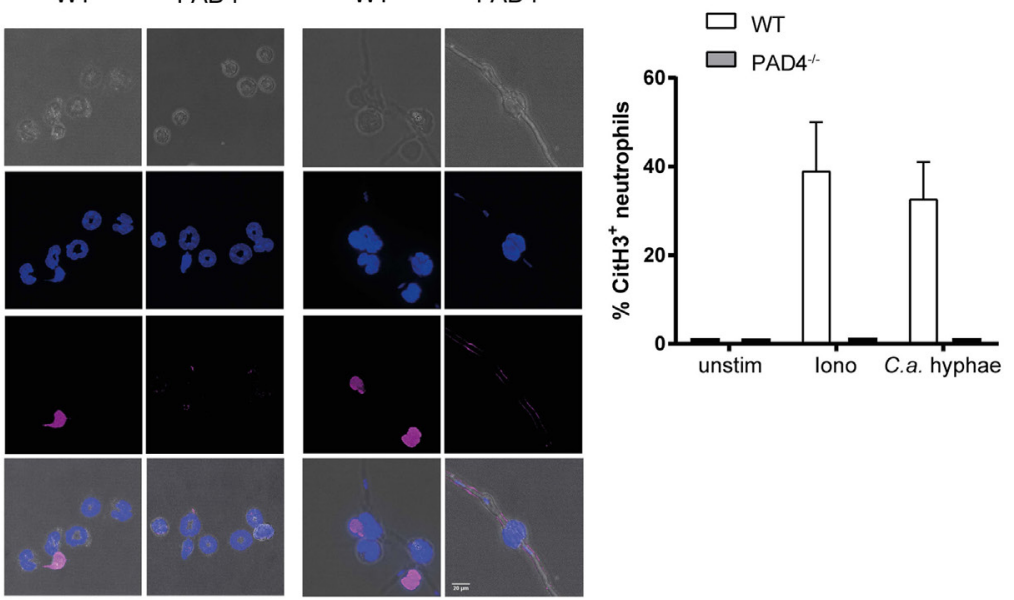

C

\section{PAS}

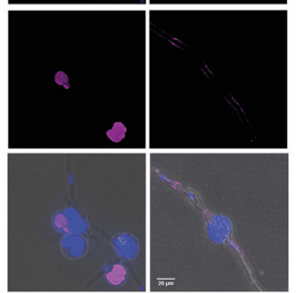

WT
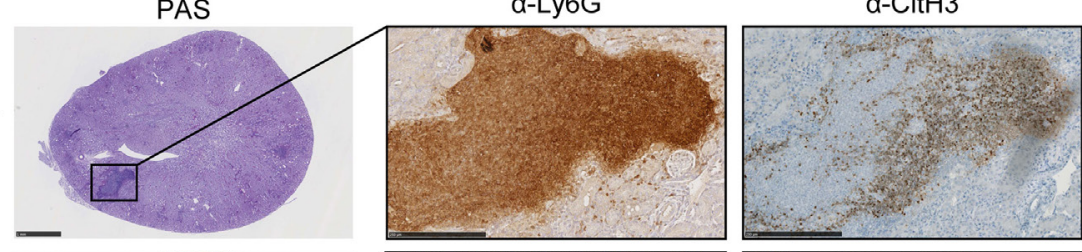

PAD4 ${ }^{-1-}$
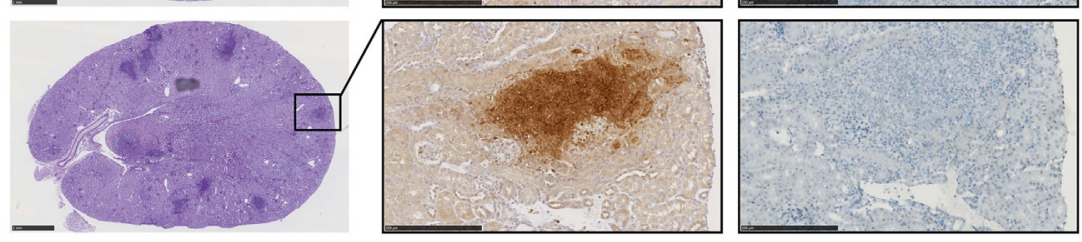

D

a-Ly6G

a-CitH3

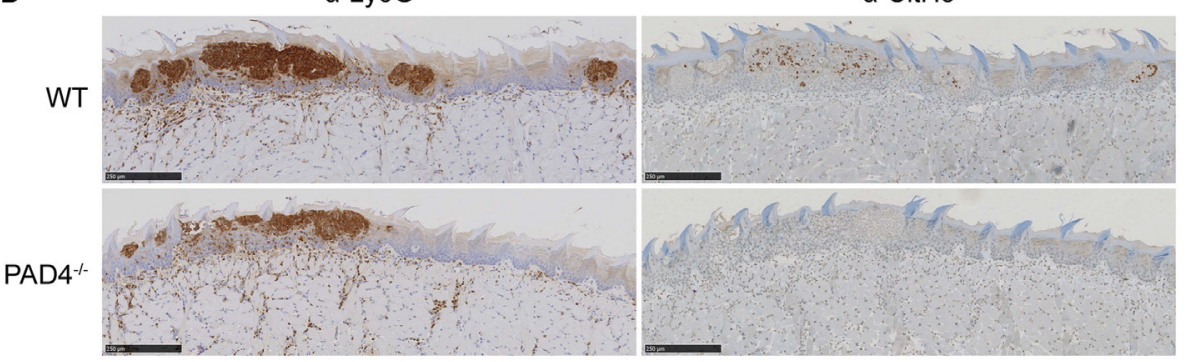

FIGURE 1 | Candida albicans induces peptidylarginine deiminase 4 (PAD4)-mediated citrullination of histone H3. (A,B) Bone marrow neutrophils from WT and $\mathrm{PAD4}^{-/-}$mice were stimulated for $2.5 \mathrm{~h}$ with $\mathrm{C}$. albicans hyphae or ionomycin or left unstimulated and then stained for DNA (DAPI; blue) and citrullinated histone H3 (magenta); brightfield. Images were acquired by confocal microscopy (A) and the fraction of $\mathrm{CitH}^{+}$neutrophils was determined (B). In (B), the bars are the mean with standard error of the mean (SEM) of 10-30 cells analyzed per condition. (C,D) WT and PAD4 $4^{-/}$mice were infected with C. albicans via the tail vein (C) or sublingually (D). Consecutive kidney (C) or tongue (D) sections were stained with periodic-acid schiff reagent (C), $\alpha$-Ly6G or with $\alpha$-CitH3 on day 3 postinfection (C) or on day 1 postinfection (D), respectively. Representative images are shown. Scale bar $=50 \mu \mathrm{m}$ [in (A)], $1 \mathrm{~mm}$ [in (C), left panel], $250 \mu \mathrm{m}$ [in (C) middle and right panel and in (D)]. See also Figure S1 in Supplementary Material. 
Material). As expected, $\mathrm{CitH} 3$ was absent in PAD4-deficient mice (Figure 1C).

Neutrophils are also implicated in antifungal defense in barrier tissues (42-44). In a model of OPC with C. albicans strain SC5314, neutrophils rapidly accumulate at sites where C. albicans hyphae invade the keratinized epithelium. They play a crucial role in preventing fungal dissemination and overgrowth within the first days of infection (43). We detected CitH3 to be induced as early as $18 \mathrm{~h}$ postinfection with a peak around $24 \mathrm{~h}$ postinfection (Figure S1C in Supplementary Material). Similarly to what we observed during systemic candidiasis, only a small fraction of all Ly6G-positive neutrophils adopted the posttranslational modification (Figure S1D in Supplementary Material) and citrullination of histone $\mathrm{H} 3$ was strictly dependent on PAD4 (Figure 1D).

\section{NETosis in Response to C. albicans Hyphae Is Independent of PAD4}

Next, we examined the role of PAD4 in NET formation in response to C. albicans hyphae. For this, we first characterized C. albicans-induced NETs in neutrophils that were isolated from the bone marrow of WT mice and stimulated with yeast cells (using the yeast-locked strain $\Delta h g c 1$ ) or with preformed hyphae (using the control strain $\Delta h g c 1+\mathrm{HGC1}$ ). NET induction was quantified by Sytox green, a DNA-intercalating dye that is impermeant to live cells (45). C. albicans hyphae triggered a strong response, which was comparable to that of ionomycin-stimulated neutrophils, while no response was observed with $C$. albicans yeast cells (Figure 2A). C. albicans hyphae-induced NETosis was independent of fungal virulence, as both, the low-virulent strain 101 (38) and the highly virulent lab strain SC5413 triggered a comparable response (Figure 2B). To confirm that the increase in Sytox fluorescence in our assay indeed corresponded to NETotic neutrophils, we stained mouse neutrophils with Sytox green for inspection of extracellular DNA by fluorescence microscopy. While unstimulated neutrophils did not bind Sytox, C. albicans hyphae induced a strong Sytox signal and Sytox ${ }^{+}$stretches of DNA extending from the cell body were observed (Figure 2C).

To assess the involvement of PAD4 in NETosis, we compared the capacity of PAD4-deficient and -sufficient neutrophils to induce NETs. Surprisingly, PAD4-deficiency did not affect the release of DNA from murine neutrophils in response to any of the stimuli tested (Figures 2A,C,D). The same was also true for human neutrophils that were treated with $\mathrm{Cl}$-amidine to inhibit $\mathrm{PAD}$ activity in response to C. albicans hyphae or PMA (Figure 2E). As a control, we included a condition with neutrophils from patients with acquired MPO-deficiency (46). As expected, these cells were strongly impaired in their capacity to release NETs in response to C. ablicans hyphae or PMA (Figure 2E).

We further compared NET release by WT and PAD4-deficient neutrophils by immunofluorescence microscopy. Upon stimulation of murine bone marrow neutrophils with C. albicans hyphae, we observed morphological changes in the plasma membrane of the cells (visualized by PKH26 staining), chromatin decondensation, and nuclear expansion characteristic of NETotic neutrophils
(Figure 3A). Moreover, we found calprotectin and MPO, which are usually located intracellularly, to colocalize with extracellular DNA (labeled with DAPI, Figure 3A). Finally, we performed scanning electron microscopy with WT and PAD $4^{-/-}$neutrophils and found NETs in proximity of C. albicans hyphae to be indistinguishable between both genotypes (Figure 3B).

In line with our previous results, we could not detect any difference in the NETs released from PAD4 ${ }^{-/}$neutrophils compared to their WT counterparts (Figure 3). Together, these data confirm that PAD4-mediated histone citrullination is dispensable for NETosis by both human and murine neutrophils in response to C. albicans hyphae.

\section{PAD4-Deficiency Results in Increased Clustering of Neutrophils Around C. albicans Hyphae but Does Not Affect Their Antifungal Activity}

Next, we investigated whether PAD4-deficiency may impact the activity of neutrophils in response to C. albicans hyphae by other means than NETosis. We observed clustering of neutrophils around fungal hyphae by fluorescence microscopy imaging. At $30 \mathrm{~min}$ of incubation, the number of neutrophils per fungal element was comparable for PAD4-deficient and -sufficient neutrophils with $\sim 2$ neutrophils per hypha (Figure 4A). At a later time point $(2.5 \mathrm{~h})$, however, the number of $\mathrm{PAD} 4^{-/}$neutrophils per hypha was increased twofold compared to WT neutrophils (Figure 4B). This result was reproducible when performing the same type of analysis with human neutrophils: inhibition of PAD4 activity by $\mathrm{Cl}$-amidine resulted in increased neutrophil numbers attached per C. albicans hyphal element (Figure 4C). We hypothesized that the increased clustering of $\mathrm{PAD}^{-/-}$neutrophils may be a consequence of an impaired ability of these cells to combat the fungus by surrounding it more densely. We, therefore, assessed by transmission electron microscopy how WT and PAD4 ${ }^{-1-}$ neutrophils affect the fungal cell wall integrity. Exposure of C. albicans hyphae to WT neutrophils caused an increase in electron density in the cell wall, compared to free C. albicans hyphae (Figure 4D). The presence of metal ions, such as copper, results in higher electron density, which is visible as dark areas in transmission electron microscopy. Thus, the increase in electron density in the cell wall may be due to enhanced expression of the copper-dependent superoxide dismutase SOD5 in C. albicans upon exposure to neutrophils (47). We observed that some hyphae displayed membrane retraction and extensive cytoplasm disintegration when incubated with neutrophils (Figure 4Di), while others presented vacuolation, nuclear alterations, and swelling of the cell wall (Figure 4Dii). We quantified the frequency of such alterations in C. albicans hyphae in response to $\mathrm{WT}$ and $\mathrm{PAD} 4^{-/-}$neutrophils. However, we did not find any evidence for a difference between the two genotypes (Figure 4E).

Likewise, PAD4-deficiency did not impair the antifungal activity of neutrophils against C. albicans. PAD $4^{-1-}$ neutrophils displayed normal capacity to phagocytose $C$. albicans yeast cells (Figure 4F) and to produce ROS in response to C. albicans 
A

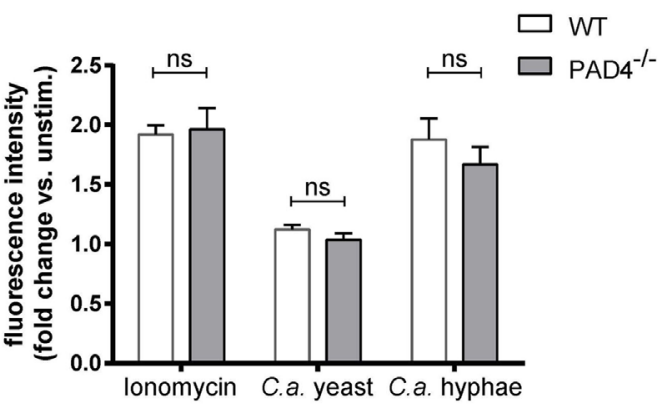

B murine neutrophils

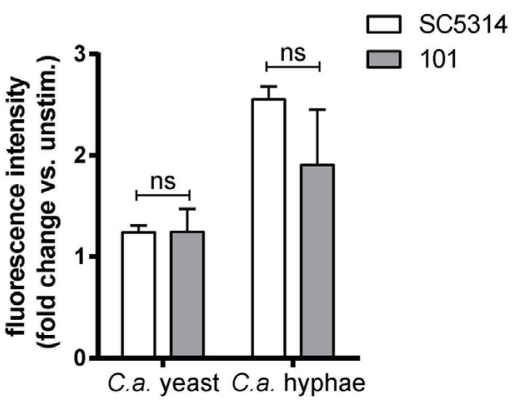

C

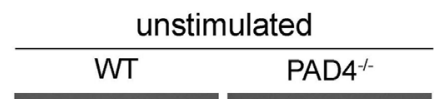

brightfield

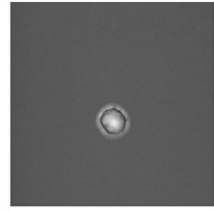

Sytox
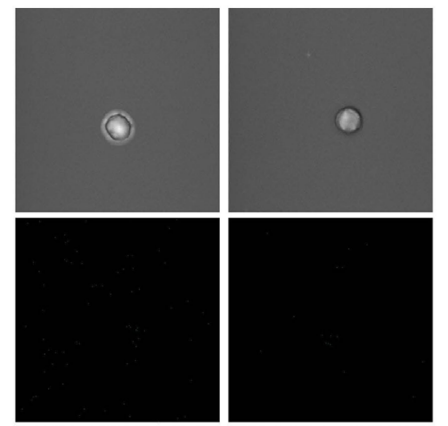

merge
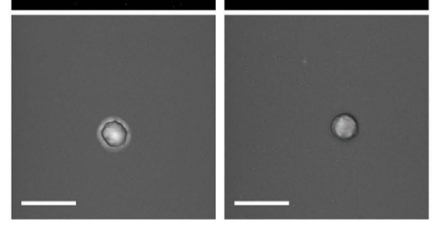

C. albicans hyphae
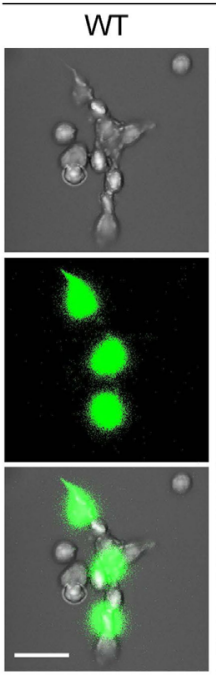

D

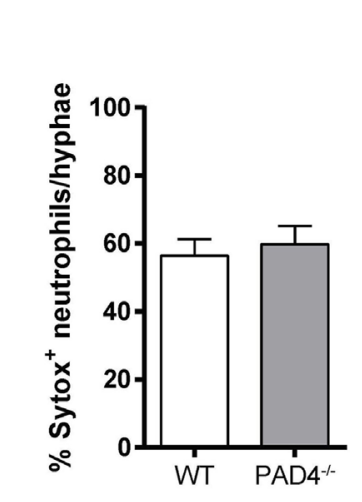

E human neutrophils
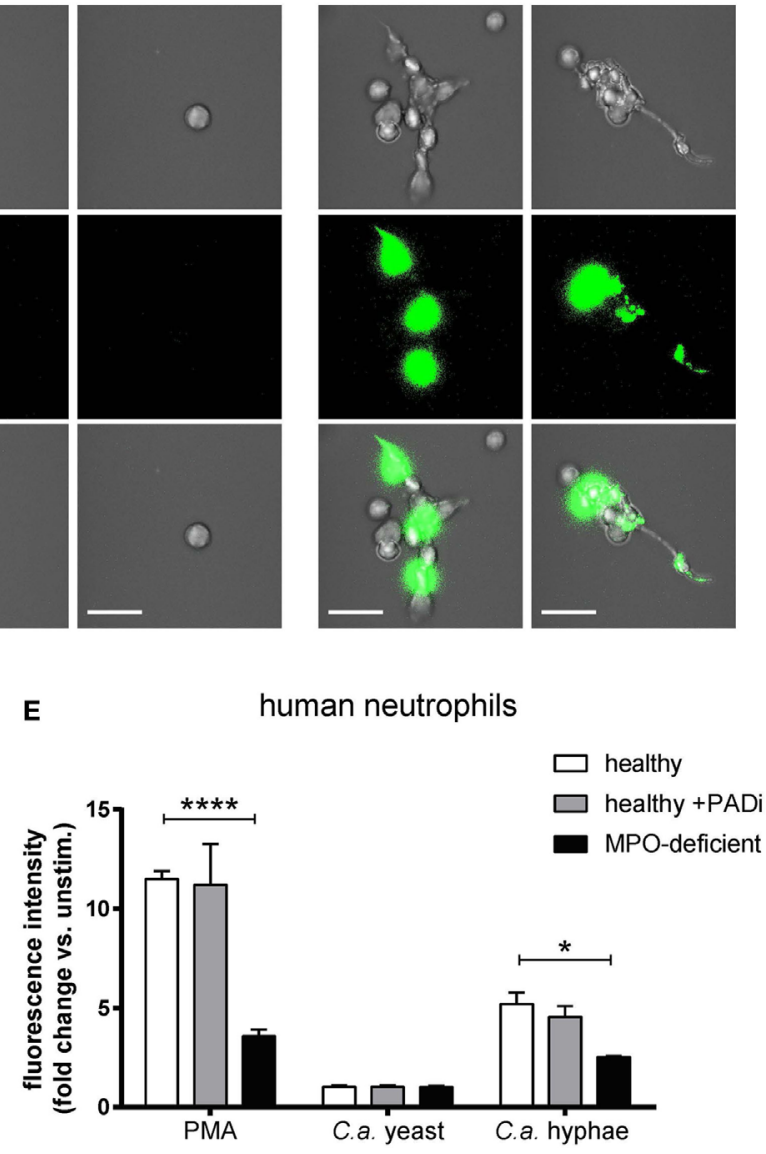

FIGURE 2 | NETosis in response to Candida albicans hyphae is independent of Peptidylarginine deiminase 4 (PAD4). (A) The release of extracellular DNA from murine bone marrow neutrophils was detected by Sytox green after stimulation for $2.5 \mathrm{~h}$ with the yeast-locked strain $\Delta h g c 1$ (C.a. yeast), preformed hyphae of the control strain $\Delta$ hgc1 + HGC1 (C.a. hyphae), or ionomycin as indicated. The increase in fluorescence intensity from stimulated relative to unstimulated neutrophils is shown. Each bar represents the mean with standard error of the mean (SEM) of each group $(n=3)$ with data pooled from three independent experiments. (B) Bone marrow neutrophils were stimulated with $C$. albicans yeast cells or preformed hyphae of the highly virulent lab strain SC5413 and the low-virulent strain 101 as indicated. The release of extracellular DNA was detected by Sytox green as in (A). Data are the mean with SEM of each group $(n=3)$ with data pooled from three independent experiments. (C,D) The release of DNA from WT and PAD4 $4^{-/}$neutrophils that were stimulated and stained with Sytox as described in (A) was visualized by immunofluorescence microscopy. Representative images for each condition are shown in (C). Scale bar $=20 \mu \mathrm{m}$. The frequency of Sytox+ neutrophils among all C. albicans hyphae-associated neutrophils is shown in (D). The bars are the mean with SEM of 15 images analyzed per condition. (E) Human peripheral blood neutrophils from healthy donors that were treated with the PAD inhibitor $\mathrm{Cl}$-amidine or left untreated and from patients with acquired myeloperoxidase-deficiency were stimulated for $2.5 \mathrm{~h}$ with the yeast-locked strain $\Delta$ hgc1 (C.a. yeast), preformed hyphae of the control strain $\Delta h g c 1+\mathrm{HGC1}$ (C.a. hyphae), or phorbol 12-myristate 13-acetate. Sytox was detected as described in (A). Each bar represents the mean with SEM of three technical replicates of each group. Data are representative of one out of two independent experiments. 


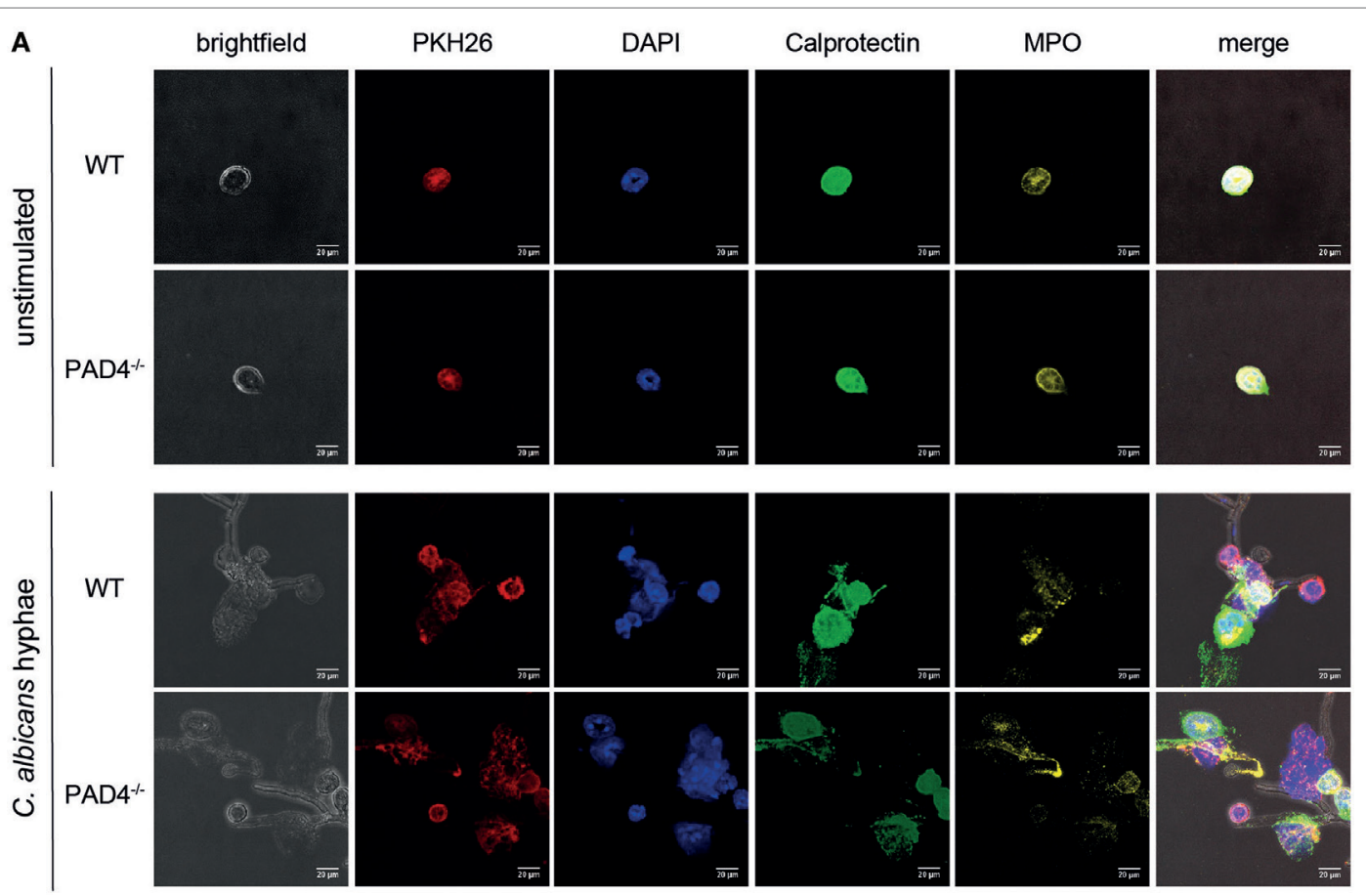

B
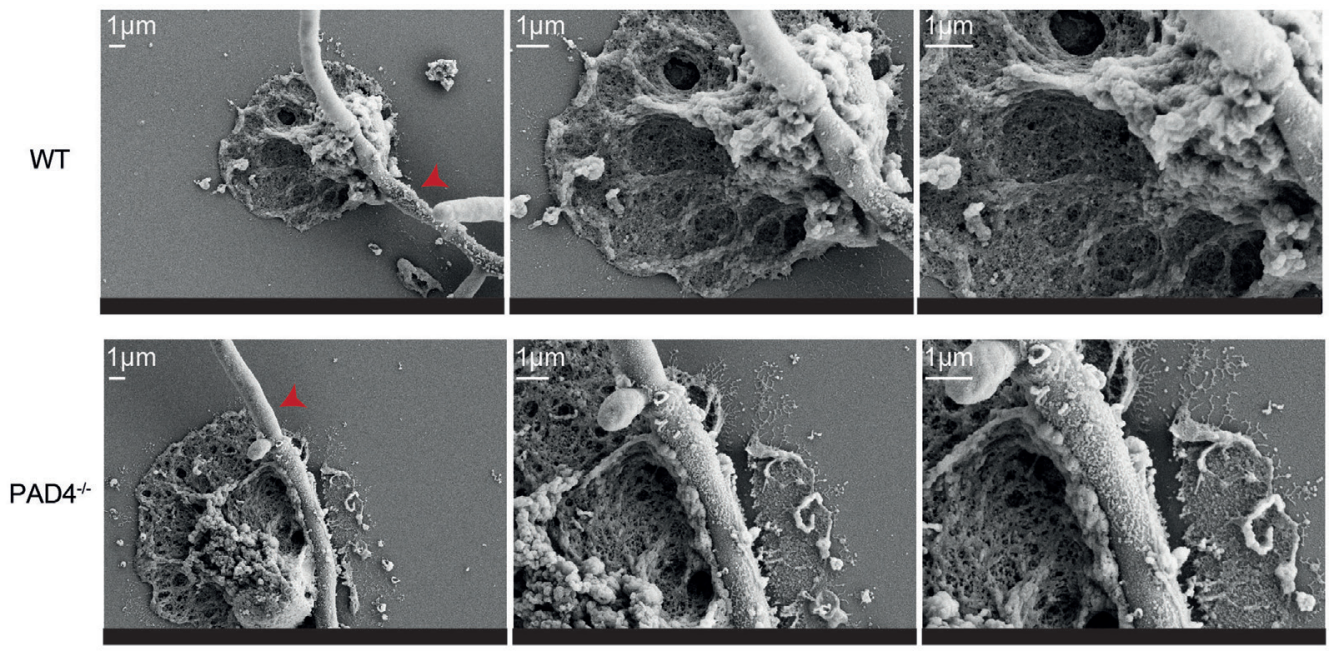

FIGURE 3 | Characterization of neutrophil extracellular traps released from WT and PAD4 ${ }^{-/-}$neutrophils. (A) Bone marrow neutrophils isolated from WT and PAD4 ${ }^{-/}$mice were stimulated for $2.5 \mathrm{~h}$ with Candida albicans hyphae or left unstimulated and then stained for membrane lipids (PKH26; red), DNA (DAPI; blue), calprotectin (anti-S100A8 and anti-S100A9, green), and myeloperoxidase (yellow). Images were acquired by confocal microscopy. Representative images of each group are shown. Scale bar $=20 \mu \mathrm{m}$. (B) Bone marrow neutrophils isolated from WT and PAD4-/- mice were stimulated for $2.5 \mathrm{~h}$ with $\mathrm{C}$. albicans hyphae and phorbol 12-myristate 13-acetate/ionomycin. Cells were fixed and imaged by scanning electron microscopy. Representative images of each group are shown. Scale bar $=1 \mu \mathrm{m}$. Red arrowheads show C. albicans hyphae.

yeast and hyphae (Figure $\mathbf{4 G}$ ). Moreover, the ability to kill C. albicans was comparable between PAD4 ${ }^{-/-}$and WT neutrophils (Figure 4H). Collectively, these results indicate that PAD4deficiency does not affect the antifungal activity of neutrophils in vitro. The meaning of the observed difference in neutrophil clustering around C. albicans hyphae could not be revealed by any of the assays performed so far, but we hypothesize that it may result in altered fungal control in vivo.

\section{PAD4 Is Not Essential for Fungal Control During Systemic and Mucosal C. albicans Infection}

To assess whether PAD4-deficiency affects fungal control in vivo, we intravenously infected $\mathrm{PAD} 4^{-/-}$mice with C. albicans. The kidney fungal burden was slightly increased in $\mathrm{PAD} 4^{-/-}$mice compared to their WT counterparts, both on day 3 and day 7 


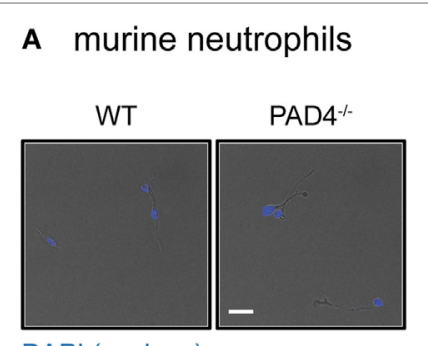

DAPI (nucleus)

B murine neutrophils

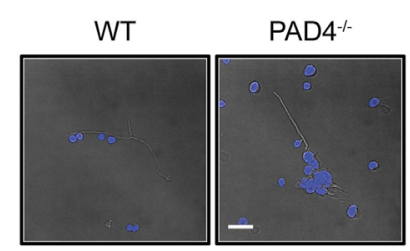

DAPI (nucleus)

C human neutrophils

Healthy donor Healthy donor

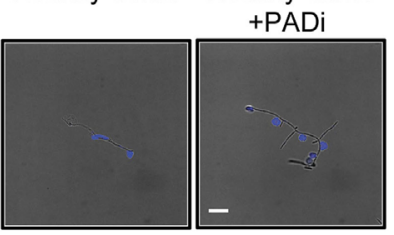

DAPI (nucleus)
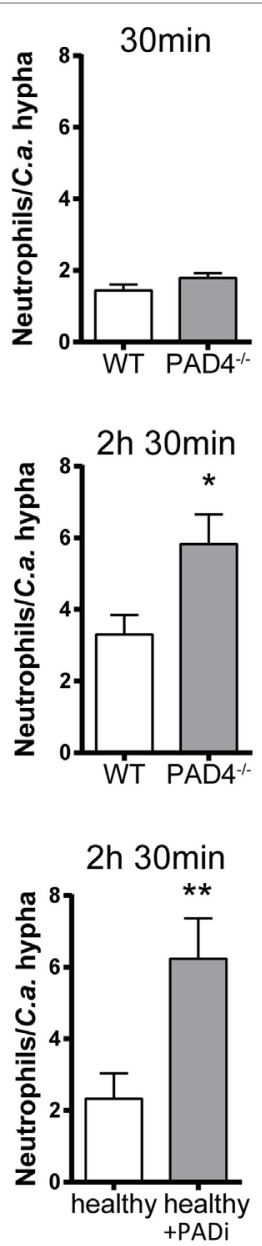

D
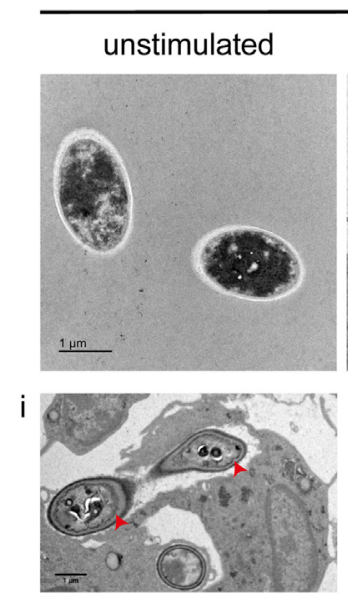

E

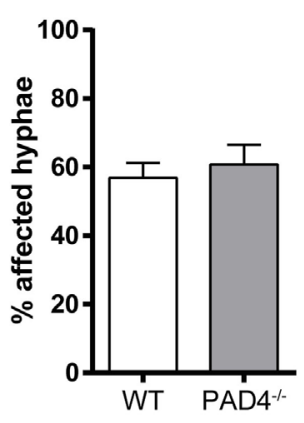

Luminol (total ROS)

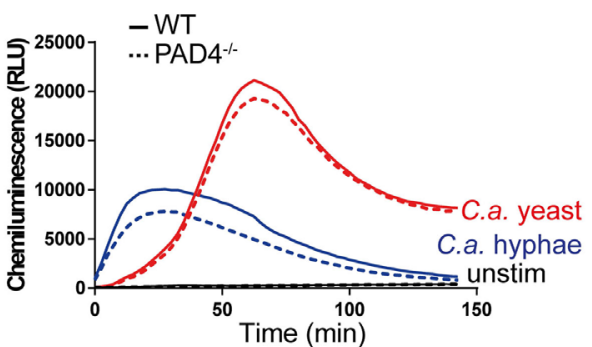

$\mathbf{F}$

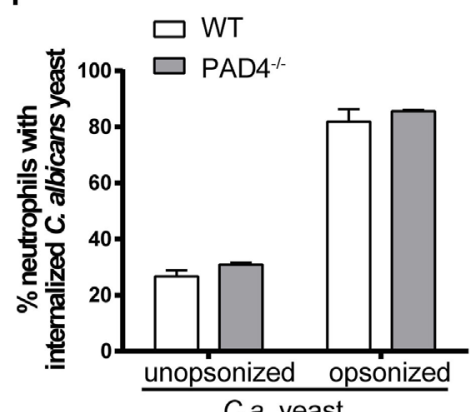

C. albicans hyphae

WT neutrophils

PAD4 $^{-/-}$neutrophils
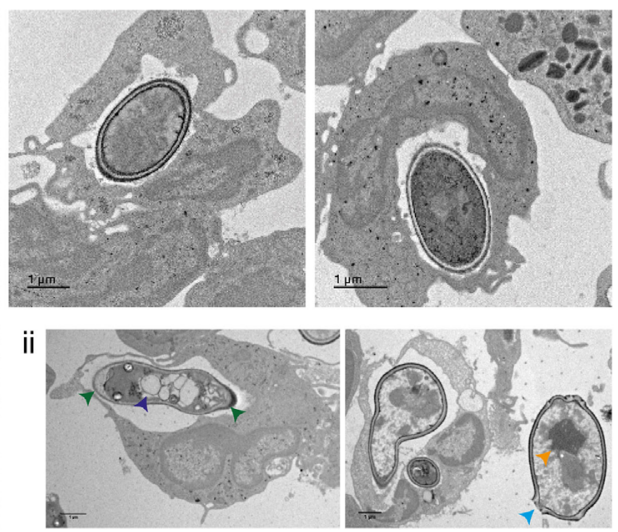

C.a. yeast

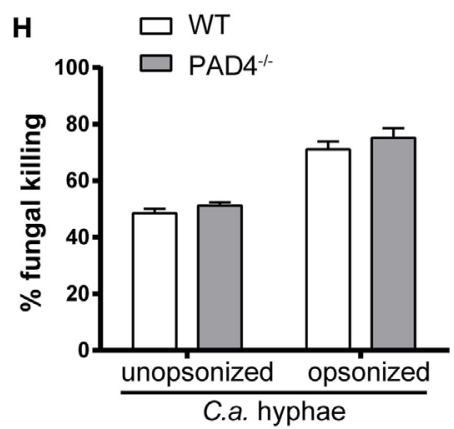

FIGURE 4 | Peptidylarginine deiminase 4 (PAD4)-deficiency results in increased clustering of neutrophils to Candida albicans hyphae but does not affect their antifungal activity. (A-C) Neutrophils were isolated from the bone marrow of naïve WT and PAD4 ${ }^{-/-}$mice $(\mathbf{A}, \mathbf{B})$ or from peripheral blood of a healthy donor and treated or not with the PAD inhibitor $\mathrm{Cl}$-amidine (C). Neutrophils were incubated with $\mathrm{C}$. albicans hyphae and then stained with DAPI (blue). The number of neutrophils interacting with each $C$. albicans hypha was quantified after 30 min (A) or $2.5 \mathrm{~h}$ (B,C) of stimulation. Representative images are shown on the left (Scale bar $=20 \mu \mathrm{m}$ ), summary plots are shown on the right. Bars are the mean with standard error of the mean (SEM) of each group with $n=2-3$. (D) The cell wall integrity of $C$. albicans hyphae was assessed by transmission electron microscopy after $2.5 \mathrm{~h}$ incubation with WT or PAD4 ${ }^{-/-}$bone marrow neutrophils.

Representative images of each group are shown in the upper row. Lower row: some hyphae exposed to neutrophils showed membrane retraction and extensive cytoplasm disintegration (Di) or vacuolation, nuclear alterations, and swelling of the cell wall (Dii). Scale bar $=1 \mu \mathrm{m}$. (E) The frequency of $C$. albicans hyphae that were affected by WT and PAD4 ${ }^{-/}$neutrophils as described in (Di,Dii) was quantified. Bars are the mean with SEM of fungal elements from 18 EM images analyzed per condition. (F) WT and PAD4 ${ }^{-/}$bone marrow neutrophils were incubated with GFP-expressing C. albicans yeast cells and the degree of Candida uptake was determined by flow cytometry. Bars are the mean with standard error of the mean (SEM) of each group with $n=2$. (G) Reactive oxygen species (ROS) production by WT and PAD4 ${ }^{-/}$bone marrow neutrophils in response to $C$. albicans yeast or hyphae was detected by chemiluminescence using the cell-impermeable substrate lucigenin to detect extracellular ROS (left) or the cell-permeable substrate luminol to detect total ROS (right). (H) WT and PAD4 ${ }^{-/-}$bone marrow neutrophils were incubated for $3 \mathrm{~h}$ with opsonized or unopsonized $C$. albicans hyphae. The percentage of $C$. albicans killing was assessed by alamar blue assay. Bars are the mean with SEM of each group with $n=4$. Data are pooled from $\mathbf{( A , B , H ) ~ o r ~ r e p r e s e n t a t i v e ~ o f ~} \mathbf{( G )}$ two independent experiments. 
postinfection (Figure 5A; Figure S2 in Supplementary Material). The increase in fungal load was accompanied by a slight increase in the number of kidney-infiltrating neutrophils (Figure 5B). However, the difference in $\mathrm{PAD} 4^{-/-}$mice compared to controls was transient, as similar fungal loads were found in the kidney of both mouse strains at a later time point of infection (Figure 5A) and the neutrophil infiltrates also swiftly normalized after the initial increase in $\mathrm{PAD}^{-/-}$mice (Figure 5B). This reversion of the initial defect in fungal control may possibly be explained by a compensatory effect of the adaptive immune system beyond
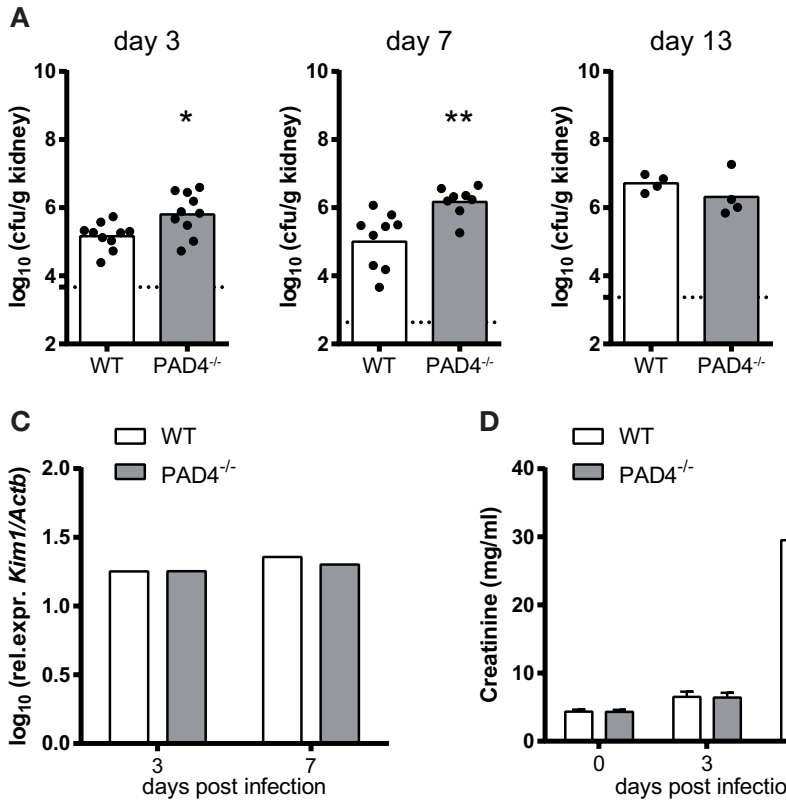

D $\square$ WT
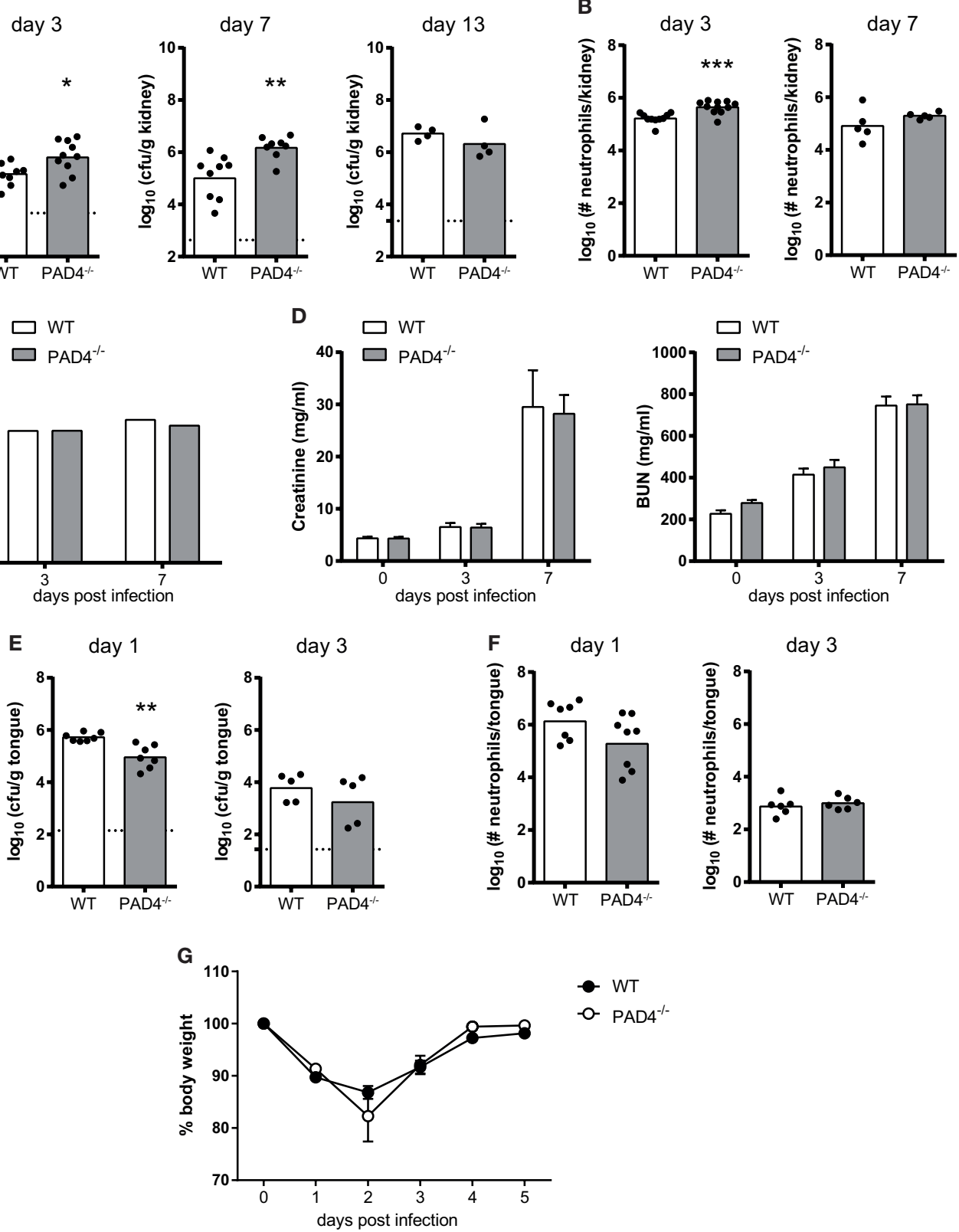

FIGURE 5 | The role of peptidylarginine deiminase 4 (PAD4) during systemic and oropharyngeal candidiasis. (A-D) WT and PAD4 ${ }^{-/-}$mice were infected with Candida albicans via the tail vein. (A) The kidney fungal burden was determined on day 3 , day 7 , and day 13 postinfection. (B) $C D 45^{+} L y 6 C^{\text {int }} L y 6 G^{+}$neutrophils were quantified in the kidney by flow cytometry on day 3 and day 7 postinfection. (C) Kidney injury molecule-1 (KIM-1) transcripts were quantified in kidney homogenates by qRT-PCR on day 3 and day 7 postinfection. (D) Creatinine and blood urea nitrogen levels were determined in the serum on day 3 and day 7 postinfection. (E-G) WT and PAD4 ${ }^{-/-}$mice were infected with $C$. albicans sublingually. (E) The tongue fungal burden was determined on day 1 and day 3 postinfection. (F) CD45+ Ly6C $C^{\text {int }} \mathrm{Ly} 6 \mathrm{G}^{+}$neutrophils were quantified in the tongue by flow cytometry on day 1 and day 3 postinfection. (G) The body weight was monitored during the course of infection. In (A,B,E,F), each symbol represents an individual mouse. The geometric mean (A-C,E,F) of each group is indicated. In (D), the bars are the mean with standard error of the mean (SEM) of each group $(n=8-10)$. Data are pooled from two to three independent experiments. The dotted lines in $\mathbf{( A , E ) ~ r e p r e s e n t ~ t h e ~ d e t e c t i o n ~ l i m i t . ~ S e e ~ a l s o ~ F i g u r e s ~ S 2 ~ a n d ~ S 3 ~ i n ~ S u p p l e m e n t a r y ~ M a t e r i a l . ~}$ 
1 week postinfection. No differences in fungal load were observed in any of the other organs that we analyzed, including brain, liver, and spleen, nor when a higher or a lower infection dose was employed (Figure S3 in Supplementary Material). We also measured kidney injury molecule-1 (KIM-1) expression and creatinine and BUN levels in C. albicans-infected mice as indicators of immunopathology. No signs of altered kidney damage (Figure 5C) or renal dysfunction (Figure 5D) were detected in $\mathrm{PAD}^{-/-}$mice compared to WT controls.

We also addressed the relevance of PAD4 in antifungal defense during mucosal infection. PAD $4^{-/-}$mice displayed normal control of the fungus in the oral cavity and tongue fungal counts were even slightly reduced at an early time point of infection compared to WT controls (Figure 5E). Neutrophil recruitment to the site of infection was not affected (Figure 5F; Figure S2 in Supplementary Material) and weight recovery after infection (Figure 5G) was also normal in $\mathrm{PAD}^{-1-}$ mice. These data indicate that PAD4 is not required for host protection against OPC, and that it has only a small and transient effect on the course of infection during systemic candidiasis.

\section{DISCUSSION}

Neutrophil extracellular traps were first described in 2004 by Brinkmann et al. as a novel mechanism used by neutrophils to combat infection by trapping and killing extracellular diseasecausing organisms including large fungal particles such as C. albicans hyphae $(12,17)$. Since then, many examples have been reported where NETs contribute to host defense and/or promote pathologies ranging from sepsis, autoinflammatory and metabolic diseases to cancer (48). NETs are released by a programed form of cell death characterized by chromatin decondensation, disassembly of the nuclear envelope, and plasma membrane disruption. NADPH oxidase, NE, and MPO play a central role in coordinating these processes (49). The protein-arginine deiminase PAD4 was also implicated in NETosis via histone citrullination and chromatin decondensation (50). In this study, we report that despite the strong induction of PAD4-dependent histone citrullination by C. albicans, this pathway is dispensable for NETosis in both mouse and human neutrophils in response to fungal hyphae. Likewise, PAD4-deficiency resulted in at best a minor and transient defect in fungal control in vivo. This was consistent with normal antifungal effector functions of PAD $4^{-/-}$neutrophils, including phagocytosis and ROS production, as previously reported (30, 34). Thus, our data challenge the paradigm that PAD4-mediated histone citrullination is essential for chromatin decondensation during NETosis.

Many activating stimuli of neutrophils can induce histone citrullination $(15,29,30,51,52)$. The observation that histone citrullination leads to chromatin decondensation associated with the release of nuclear DNA (29) led to the assumption that PAD4 activity is an integral part of the NETotic process (50). However, the requirement of PAD4 in NETosis was never formally proven and although the link between histone citrullination and NETosis is widely accepted it remains at best correlative. The excitement about the availability of an allegedly specific marker of NETosis has drowned critical voices questioning the erroneous concept of citrullination in NETosis $(33,53,54)$. Our study now demonstrates that PAD4-mediated histone citrullination is uncoupled from NETosis.

NETosis is induced by diverse stimuli. In this study, we focused on the fungal pathogen C. albicans, a prominent inducer of NETosis $(13,15)$, and we also included the non-microbial stimuli PMA and ionomycin. Because of the $\mathrm{Ca}^{2+}$-dependency of PAD4 activity (31), $\mathrm{Ca}^{2+}$ ionophores were postulated to act as bona fide stimuli for PAD4-mediated histone citrullination linked to subsequent chromatin release (53). In our hands, however, NET induction by ionomycin also remained unaffected by PAD4-inhibition. We, therefore, believe that the independence of NETosis from histone citrullination is broadly applicable, although we cannot exclude stimulus-dependent involvement of PAD4 in NETosis.

Diverse processes have been attributed to PAD4 activity beyond NETosis. Although histones were among the first substrates of PAD4 to be identified $(55,56)$, over 70 putative PAD4 substrates have been described to date $(57,58)$. PAD4 activity is a general hallmark of neutrophil activation. In addition to modifying histones, PAD4 citrullinates transcription factors including $\mathrm{NF \kappa B}$ and $\mathrm{E} 2 \mathrm{~F}-1$ for enhanced nuclear translocation or promoter binding, respectively, and increased cytokine production $(59,60)$. PAD4 can also modulate $\mathrm{T}$ cell-mediated immune responses $(61,62)$ and the activity of inflammatory mediators including cytokines, chemokines, and host defense proteins (63-66), among other reported functions. Any of these NET-independent activities of PAD4 may underlie the slight defect in fungal control that we observed in PAD4-deficient mice during systemic candidiasis.

The implication of citrullinated autoantigens including citrullinated histones in rheumatoid arthritis led to the idea that NETosis is a source of these autoantigens and contributes to disease pathogenesis (67-69). Based on the above considerations, however, the role of PAD4 in rheumatoid arthritis is more likely independent of NETosis. Likewise, the idea that deletion or inhibition of PAD4 improves disease symptoms in preclinical models of lupus, vasculitis, and glomerulonephritis due to inhibition of NETosis (70-72) calls for careful re-analysis. The pharmacological targeting of PAD4 in these diseases in human patients should be approached with care before fully understanding the pathomechanism in each condition. Moreover, another member of the peptidylarginine deiminase family, PAD2, can also catalyze protein citrullination in response to certain stimuli including TNF (73).

Citrullination of histones can easily be detected by immunohistochemistry or immunofluorescence on tissue sections. The postulated link between PAD4-mediated histone citrullination and NETosis introduced a welcome approach for easy identification of NETs and, consequently, this posttranslational modification is now commonly used as a proxy for NETs, especially in tissues in vivo $(15,30,35,73,74)$. Based on our results, however, histone citrullination can no longer be accepted as a reliable marker for NETs. 
Direct visualization of NETs remains difficult, especially in tissues where a high cell density and autofluorescence hinders the unambiguous extracellular localization of DNA and proteins. Accordingly, we were unable to detect extracellular chromosomal DNA in the C. albicans-infected kidney or tongue, both organs displaying a particularly high cell density and high autofluorescence. To overcome these tissue-specific limitations, most in vivo studies on NETs focused on the lung or the vasculature $(13,15,75)$. However, these are not target organs of C. albicans. A specific marker for the visualization of NETs is still awaiting its discovery.

The lack of a specific marker for NETs has also hampered the assessment of the requirement of NETs in various conditions. PAD4-deficient mice were employed as a model of NETdeficiency to test the antimicrobial role of NETs in immune defense against various pathogenic microbes, including Shigella flexneri, group A Streptococcus, and influenza virus (30, 34, 35). Only in some cases an impairment in the protective response was observed, which was attributed to defects in NETosis (30), while susceptibility to infection was not generally affected by PAD4-deficiency $(34,35)$. Our findings put in question the interpretation of the results from studies with PAD4-deficient mice, as we show that NETosis occurs independently of PAD4.

Lytic and non-lytic forms of NETosis have been described. The latter is independent of NADPH oxidase and MPO and does not involve citrullination of histone H3 (76). Although we cannot formally exclude a non-lytic mechanism of NETosis as an alternative explanation for PAD4-independent NETosis in response to C. albicans, our data with neutrophils from acquired MPO-deficiency and the visualization of chromatin decondensation support a lytic process.

The distinction of NETosis from other forms of lytic cell death that are accompanied with the release of DNA into the extracellular space such as necroptosis, which can occur as a consequence of cellular damage, remains difficult (77). Bacteria such as Staphylococcus aureus can induce NET-like structures via pore-forming toxins that induce $\mathrm{Ca}^{2+}$ influx, citrullination, and chromatin extrusion $(78,79)$. Moreover, particles of different sizes and shapes have been shown to induce NET-like DNA release in association with neutrophil necroptosis, both in human and murine neutrophils (80). Whether C. albicans-induced NETlike structures result from cellular damage caused by hyphal virulence factors has not been fully clarified. C. albicans-secreted aspartyl proteinases seem to play an important role in triggering NETosis (81). Whether the recently identified pore-forming toxin Candidalysin, which can induce cellular damage in host cells (82), also contributes to NETosis has not been reported. While research on Candidalysin focused mainly on epithelial cells, Candidalysin may also cause damage in immune cells (83) including neutrophils. We found NETs to be induced by different isolates of C. albicans including strain 101, which expresses only very low levels of the Candidalysin-encoding gene ECE1 and is unable to cause cellular damage (38). This result contradicts the idea that C. albicans-induced NETosis may be the result of toxin-induced cellular damage. More work is needed to identify the fungal determinants and the neutrophil molecular pathways sensing C. albicans for NET induction.

Together, our study confirms the efficient induction of NETosis by C. albicans hyphae, but it challenges the current view on the molecular mechanism of NET induction as well as the reliability of frequently used approaches in the field to document the generation and function of NETs in vivo.

\section{ETHICS STATEMENT}

All mouse experiments described in this study were conducted in strict accordance with the guidelines of the Swiss Animal Protection Law and were performed under protocols approved by the Veterinary office of the Canton Zurich, Switzerland (license number 201/2012 and 183/2015). All efforts were made to minimize suffering and ensure the highest ethical and humane standards. Experiments with human blood samples were approved by the local ethics committee (KEK-ZH-NR: 20090062, BASEC-Nr. 2016-01908) and were performed and analyzed anonymously. Blood samples were obtained after informed consent and in accordance with the declaration of Helsinki principles.

\section{AUTHOR CONTRIBUTIONS}

EG and SL-L designed the experiments and wrote the manuscript. EG, CL, and NK performed experiments. EG, CL, NK, and SL-L analyzed data. ES performed transmission electron microscopy. AT provided peripheral blood samples from patients with acquired MPO-deficiency. SL-L supervised the project.

\section{ACKNOWLEDGMENTS}

The authors would like to thank Maries van den Broek for mice; Constantin Urban for S100A8 and S100A9 antibodies; Sarah Mertens for technical assistance; the staff of Laboratory Animal Service Center of the University of Zürich for animal husbandry; Giovanni Pellegrini from the Laboratory for Animal Model Pathology of the University of Zürich for expertise in animal pathology; the Center for Microscopy and Image Analysis of the University of Zürich for scanning electron microscopy; Annette Oxenius, Onur Boyman, and all members of the LeibundGut-lab for helpful advice and discussions.

\section{FUNDING}

This work was supported by an ETH research grant (ETH-26 14-1), the Swiss National Science Foundation (310030-166206) and University of Zürich.

\section{SUPPLEMENTARY MATERIAL}

The Supplementary Material for this article can be found online at https://www.frontiersin.org/articles/10.3389/fimmu.2018.01573/ full\#supplementary-material. 


\section{REFERENCES}

1. Brown GD, Denning DW, Gow NA, Levitz SM, Netea MG, White TC. Hidden killers: human fungal infections. Sci Transl Med (2012) 4:165rv13. doi:10.1126/scitranslmed.3004404

2. Noble SM, Gianetti BA, Witchley JN. Candida albicans cell-type switching and functional plasticity in the mammalian host. Nat Rev Microbiol (2017) 15:96-108. doi:10.1038/nrmicro.2016.157

3. Berman J, Sudbery PE. Candida albicans: a molecular revolution built on lessons from budding yeast. Nat Rev Genet (2002) 3:918-30. doi:10.1038/ $\operatorname{nrg} 948$

4. Jacobsen ID, Wilson D, Wachtler B, Brunke S, Naglik JR, Hube B. Candida albicans dimorphism as a therapeutic target. Expert Rev Anti Infect Ther (2012) 10:85-93. doi:10.1586/eri.11.152

5. Zhu W, Filler SG. Interactions of Candida albicans with epithelial cells. Cell Microbiol (2010) 12:273-82. doi:10.1111/j.1462-5822.2009.01412.x

6. d'Enfert C. Hidden killers: persistence of opportunistic fungal pathogens in the human host. Curr Opin Microbiol (2009) 12:358-64. doi:10.1016/j. mib.2009.05.008

7. Brown GD. Innate antifungal immunity: the key role of phagocytes. Annu Rev Immunol (2011) 29:1-21. doi:10.1146/annurev-immunol-030409101229

8. Pfaller MA, Diekema DJ. Epidemiology of invasive candidiasis: a persistent public health problem. Clin Microbiol Rev (2007) 20:133-63. doi:10.1128/ CMR.00029-06

9. Fulurija A, Ashman RB, Papadimitriou JM. Neutrophil depletion increases susceptibility to systemic and vaginal candidiasis in mice, and reveals differences between brain and kidney in mechanisms of host resistance. Microbiology (1996) 142:3487-96. doi:10.1099/13500872-142-12-3487

10. Lionakis MS, Fischer BG, Lim JK, Swamydas M, Wan W, Richard Lee CC, et al. Chemokine receptor Ccrl drives neutrophil-mediated kidney immunopathology and mortality in invasive candidiasis. PLoS Pathog (2012) 8:e1002865. doi:10.1371/journal.ppat.1002865

11. Majer O, Bourgeois C, Zwolanek F, Lassnig C, Kerjaschki D, Mack M, et al. Type I interferons promote fatal immunopathology by regulating inflammatory monocytes and neutrophils during Candida infections. PLoS Pathog (2012) 8:e1002811. doi:10.1371/journal.ppat.1002811

12. Brinkmann V, Reichard U, Goosmann C, Fauler B, Uhlemann Y, Weiss DS, et al. Neutrophil extracellular traps kill bacteria. Science (2004) 303:1532-5. doi:10.1126/science. 1092385

13. Urban CF, Ermert D, Schmid M, Abu-Abed U, Goosmann C, Nacken W, et al. Neutrophil extracellular traps contain calprotectin, a cytosolic protein complex involved in host defense against Candida albicans. PLoS Pathog (2009) 5:e1000639. doi:10.1371/journal.ppat.1000639

14. Erwig LP, Gow NA. Interactions of fungal pathogens with phagocytes. Nat Rev Microbiol (2016) 14:163-76. doi:10.1038/nrmicro.2015.21

15. Branzk N, Lubojemska A, Hardison SE, Wang Q, Gutierrez MG, Brown GD, et al. Neutrophils sense microbe size and selectively release neutrophil extracellular traps in response to large pathogens. Nat Immunol (2014) 15:1017-25. doi:10.1038/ni.2987

16. Brinkmann V, Zychlinsky A. Neutrophil extracellular traps: is immunity the second function of chromatin? J Cell Biol (2012) 198:773-83. doi:10.1083/ jcb. 201203170

17. Urban CF, Reichard U, Brinkmann V, Zychlinsky A. Neutrophil extracellular traps capture and kill Candida albicans yeast and hyphal forms. Cell Microbiol (2006) 8:668-76. doi:10.1111/j.1462-5822.2005.00659.x

18. Abi Abdallah DS, Lin C, Ball CJ, King MR, Duhamel GE, Denkers EY. Toxoplasma gondii triggers release of human and mouse neutrophil extracellular traps. Infect Immun (2012) 80:768-77. doi:10.1128/IAI.05730-11

19. Delgado-Rizo V, Martinez-Guzman MA, Iniguez-Gutierrez L, Garcia-Orozco A, Alvarado-Navarro A, Fafutis-Morris M. Neutrophil extracellular traps and its implications in inflammation: an overview. Front Immunol (2017) 8:81. doi:10.3389/fimmu.2017.00081

20. Kolaczkowska E, Kubes P. Neutrophil recruitment and function in health and inflammation. Nat Rev Immunol (2013) 13:159-75. doi:10.1038/nri3399

21. Wartha F, Beiter K, Normark S, Henriques-Normark B. Neutrophil extracellular traps: casting the NET over pathogenesis. Curr Opin Microbiol (2007) 10:52-6. doi:10.1016/j.mib.2006.12.005
22. Fuchs TA, Brill A, Duerschmied D, Schatzberg D, Monestier M, Myers DD Jr, et al. Extracellular DNA traps promote thrombosis. Proc Natl Acad Sci U S A (2010) 107:15880-5. doi:10.1073/pnas.1005743107

23. Kessenbrock K, Krumbholz M, Schonermarck U, Back W, Gross WL, Werb Z, et al. Netting neutrophils in autoimmune small-vessel vasculitis. Nat Med (2009) 15:623-5. doi:10.1038/nm.1959

24. Lin AM, Rubin CJ, Khandpur R, Wang JY, Riblett M, Yalavarthi S, et al. Mast cells and neutrophils release IL-17 through extracellular trap formation in psoriasis. J Immunol (2011) 187:490-500. doi:10.4049/jimmunol.1100123

25. Cools-Lartigue J, Spicer J, McDonald B, Gowing S, Chow S, Giannias B, et al. Neutrophil extracellular traps sequester circulating tumor cells and promote metastasis. J Clin Invest (2013). doi:10.1172/JCI67484

26. Fuchs TA, Abed U, Goosmann C, Hurwitz R, Schulze I, Wahn V, et al. Novel cell death program leads to neutrophil extracellular traps. J Cell Biol (2007) 176:231-41. doi:10.1083/jcb.200606027

27. Metzler KD, Fuchs TA, Nauseef WM, Reumaux D, Roesler J, Schulze I, et al. Myeloperoxidase is required for neutrophil extracellular trap formation: implications for innate immunity. Blood (2011) 117:953-9. doi:10.1182/ blood-2010-06-290171

28. Papayannopoulos V, Metzler KD, Hakkim A, Zychlinsky A. Neutrophil elastase and myeloperoxidase regulate the formation of neutrophil extracellular traps. J Cell Biol (2010) 191:677-91. doi:10.1083/jcb.201006052

29. Wang Y, Li M, Stadler S, Correll S, Li P, Wang D, et al. Histone hypercitrullination mediates chromatin decondensation and neutrophil extracellular trap formation. J Cell Biol (2009) 184:205-13. doi:10.1083/jcb.200806072

30. Li P, Li M, Lindberg MR, Kennett MJ, Xiong N, Wang Y. PAD4 is essential for antibacterial innate immunity mediated by neutrophil extracellular traps. J Exp Med (2010) 207:1853-62. doi:10.1084/jem.20100239

31. Vossenaar ER, Zendman AJ, van Venrooij WJ, Pruijn GJ. PAD, a growing family of citrullinating enzymes: genes, features and involvement in disease. Bioessays (2003) 25:1106-18. doi:10.1002/bies.10357

32. Baka Z, Gyorgy B, Geher P, Buzas EI, Falus A, Nagy G. Citrullination under physiological and pathological conditions. Joint Bone Spine (2012) 79:431-6. doi:10.1016/j.jbspin.2012.01.008

33. Kenny EF, Herzig A, Kruger R, Muth A, Mondal S, Thompson PR, et al. Diverse stimuli engage different neutrophil extracellular trap pathways. Elife (2017) 6:e24437. doi:10.7554/eLife.24437

34. Martinod K, Fuchs TA, Zitomersky NL, Wong SL, Demers M, Gallant M, et al. PAD4-deficiency does not affect bacteremia in polymicrobial sepsis and ameliorates endotoxemic shock. Blood (2015) 125:1948-56. doi:10.1182/ blood-2014-07-587709

35. Hemmers S, Teijaro JR, Arandjelovic S, Mowen KA. PAD4-mediated neutrophil extracellular trap formation is not required for immunity against influenza infection. PLoS One (2011) 6:e22043. doi:10.1371/journal.pone. 0022043

36. Gillum AM, Tsay EY, Kirsch DR. Isolation of the Candida albicans gene for orotidine-5'-phosphate decarboxylase by complementation of S. cerevisiae ura3 and E. coli pyrF mutations. Mol Gen Genet (1984) 198:179-82. doi:10.1007/ BF00328721

37. Zheng X, Wang Y, Wang Y. Hgc1, a novel hypha-specific G1 cyclin-related protein regulates Candida albicans hyphal morphogenesis. EMBO J (2004) 23:1845-56. doi:10.1038/sj.emboj.7600195

38. Schonherr FA, Sparber F, Kirchner FR, Guiducci E, Trautwein-Weidner K, Gladiator A, et al. The intraspecies diversity of C. albicans triggers qualitatively and temporally distinct host responses that determine the balance between commensalism and pathogenicity. Mucosal Immunol (2017) 10:1335-50. doi: $10.1038 / \mathrm{mi} .2017 .2$

39. Barelle CJ, Manson CL, MacCallum DM, Odds FC, Gow NA, Brown AJ. GFP as a quantitative reporter of gene regulation in Candida albicans. Yeast (2004) 21:333-40. doi:10.1002/yea.1099

40. Solis NV, Filler SG. Mouse model of oropharyngeal candidiasis. Nat Protoc (2012) 7:637-42. doi:10.1038/nprot.2012.011

41. Lionakis MS, Lim JK, Lee CC, Murphy PM. Organ-specific innate immune responses in a mouse model of invasive candidiasis. J Innate Immun (2011) 3:180-99. doi:10.1159/000321157

42. Huppler AR, Conti HR, Hernandez-Santos N, Darville T, Biswas PS, Gaffen SL. Role of neutrophils in IL-17-dependent immunity to mucosal candidiasis. J Immunol (2014) 192:1745-52. doi:10.4049/jimmunol.1302265 
43. Trautwein-Weidner K, Gladiator A, Nur S, Diethelm P, LeibundGut-Landmann S. IL-17-mediated antifungal defense in the oral mucosa is independent of neutrophils. Mucosal Immunol (2015) 8:221-31. doi:10.1038/mi.2014.57

44. Altmeier S, Toska A, Sparber F, Teijeira A, Halin C, LeibundGut-Landmann S. IL-1 coordinates the neutrophil response to C. albicans in the oral mucosa. PLoS Pathog (2016) 12:e1005882. doi:10.1371/journal.ppat.1005882

45. de Buhr N, von Kockritz-Blickwede M. How neutrophil extracellular traps become visible. J Immunol Res (2016) 2016:4604713. doi:10.1155/2016/ 4604713

46. Theocharides AP, Lundberg P, Lakkaraju AK, Lysenko V, Myburgh R, Aguzzi A, et al. Homozygous calreticulin mutations in patients with myelofibrosis lead to acquired myeloperoxidase deficiency. Blood (2016) 127:3253-9. doi:10.1182/blood-2016-02-696310

47. Gleason JE, Galaleldeen A, Peterson RL, Taylor AB, Holloway SP, WaningerSaroni J, et al. Candida albicans SOD5 represents the prototype of an unprecedented class of $\mathrm{Cu}$-only superoxide dismutases required for pathogen defense. Proc Natl Acad Sci U S A (2014) 111:5866-71. doi:10.1073/pnas. 1400137111

48. Jorch SK, Kubes P. An emerging role for neutrophil extracellular traps in noninfectious disease. Nat Med (2017) 23:279-87. doi:10.1038/nm.4294

49. Papayannopoulos V. Neutrophil extracellular traps in immunity and disease. Nat Rev Immunol (2018) 18:134-47. doi:10.1038/nri.2017.105

50. Rohrbach AS, Slade DJ, Thompson PR, Mowen KA. Activation of PAD4 in NET formation. Front Immunol (2012) 3:360. doi:10.3389/fimmu.2012.00360

51. Warnatsch A, Ioannou M, Wang Q, Papayannopoulos V. Inflammation. Neutrophil extracellular traps license macrophages for cytokine production in atherosclerosis. Science (2015) 349:316-20. doi:10.1126/science.aaa8064

52. Hosseinzadeh A, Thompson PR, Segal BH, Urban CF. Nicotine induces neutrophil extracellular traps. J Leukoc Biol (2016) 100:1105-12. doi:10.1189/ jlb.3AB0815-379RR

53. Konig MF, Andrade F. A critical reappraisal of neutrophil extracellular traps and NETosis mimics based on differential requirements for protein citrullination. Front Immunol (2016) 7:461. doi:10.3389/fimmu. 2016.00461

54. Neeli I, Radic M. Opposition between PKC isoforms regulates histone deimination and neutrophil extracellular chromatin release. Front Immunol (2013) 4:38. doi:10.3389/fimmu.2013.00038

55. Nakashima K, Hagiwara T, Yamada M. Nuclear localization of peptidylarginine deiminase $\mathrm{V}$ and histone deimination in granulocytes. J Biol Chem (2002) 277:49562-8. doi:10.1074/jbc.M208795200

56. Hagiwara T, Nakashima K, Hirano H, Senshu T, Yamada M. Deimination of arginine residues in nucleophosmin/B23 and histones in HL-60 granulocytes. Biochem Biophys Res Commun (2002) 290:979-83. doi:10.1006/ bbrc.2001.6303

57. Guo Q, Bedford MT, Fast W. Discovery of peptidylarginine deiminase-4 substrates by protein array: antagonistic citrullination and methylation of human ribosomal protein S2. Mol Biosyst (2011) 7:2286-95. doi:10.1039/ clmb05089c

58. Assohou-Luty C, Raijmakers R, Benckhuijsen WE, Stammen-Vogelzangs J, de Ru A, van Veelen PA, et al. The human peptidylarginine deiminases type 2 and type 4 have distinct substrate specificities. Biochim Biophys Acta (2014) 1844:829-36. doi:10.1016/j.bbapap.2014.02.019

59. Ghari F, Quirke AM, Munro S, Kawalkowska J, Picaud S, McGouran J, et al. Citrullination-acetylation interplay guides E2F-1 activity during the inflammatory response. Sci $A d v$ (2016) 2:e1501257. doi:10.1126/sciadv.1501257

60. Sun B, Dwivedi N, Bechtel TJ, Paulsen JL, Muth A, Bawadekar M, et al. Citrullination of NF-kappaB p65 promotes its nuclear localization and TLR-induced expression of IL-1beta and TNFalpha. Sci Immunol (2017) 2. doi:10.1126/sciimmunol.aal3062

61. Kawalkowska J, Quirke AM, Ghari F, Davis S, Subramanian V, Thompson PR, et al. Abrogation of collagen-induced arthritis by a peptidyl arginine deiminase inhibitor is associated with modulation of T cell-mediated immune responses. Sci Rep (2016) 6:26430. doi:10.1038/srep26430

62. Jang B, Kim HW, Kim JS, Kim WS, Lee BR, Kim S, et al. Peptidylarginine deiminase inhibition impairs toll-like receptor agonist-induced functional maturation of dendritic cells, resulting in the loss of T cell-proliferative capacity: a partial mechanism with therapeutic potential in inflammatory settings. J Leukoc Biol (2015) 97:351-62. doi:10.1189/jlb.3A0314$142 \mathrm{RR}$
63. Moelants EA, Van Damme J, Proost P. Detection and quantification of citrullinated chemokines. PLoS One (2011) 6:e28976. doi:10.1371/journal. pone. 0028976

64. Moelants EA, Mortier A, Grauwen K, Ronsse I, Van Damme J, Proost P. Citrullination of TNF-alpha by peptidylarginine deiminases reduces its capacity to stimulate the production of inflammatory chemokines. Cytokine (2013) 61:161-7. doi:10.1016/j.cyto.2012.09.011

65. Loos T, Mortier A, Gouwy M, Ronsse I, Put W, Lenaerts JP, et al. Citrullination of CXCL10 and CXCL11 by peptidylarginine deiminase: a naturally occurring posttranslational modification of chemokines and new dimension of immunoregulation. Blood (2008) 112:2648-56. doi:10.1182/blood2008-04-149039

66. Koziel J, Bryzek D, Sroka A, Maresz K, Glowczyk I, Bielecka E, et al. Citrullination alters immunomodulatory function of LL-37 essential for prevention of endotoxin-induced sepsis. J Immunol (2014) 192:5363-72. doi:10.4049/jimmunol.1303062

67. Spengler J, Lugonja B, Ytterberg AJ, Zubarev RA, Creese AJ, Pearson MJ, et al. Release of active peptidyl arginine deiminases by neutrophils can explain production of extracellular citrullinated autoantigens in rheumatoid arthritis synovial fluid. Arthritis Rheumatol (2015) 67:3135-45. doi:10.1002/art.39313

68. Corsiero E, Bombardieri M, Carlotti E, Pratesi F, Robinson W, Migliorini P, et al. Single cell cloning and recombinant monoclonal antibodies generation from RA synovial B cells reveal frequent targeting of citrullinated histones of NETs. Ann Rheum Dis (2016) 75:1866-75. doi:10.1136/annrheumdis2015-208356

69. Chang HH, Dwivedi N, Nicholas AP, Ho IC. The W620 polymorphism in PTPN22 disrupts its interaction with peptidylarginine deiminase type 4 and enhances citrullination and NETosis. Arthritis Rheumatol (2015) 67:2323-34. doi:10.1002/art.39215

70. Knight JS, Subramanian V, O'Dell AA, Yalavarthi S, Zhao W, Smith CK, et al. Peptidylarginine deiminase inhibition disrupts NET formation and protects against kidney, skin and vascular disease in lupus-prone MRL/lpr mice. Ann Rheum Dis (2015) 74:2199-206. doi:10.1136/annrheumdis-2014-205365

71. Kumar SV, Kulkarni OP, Mulay SR, Darisipudi MN, Romoli S, Thomasova D, et al. Neutrophil extracellular trap-related extracellular histones cause vascular necrosis in severe GN. J Am Soc Nephrol (2015) 26:2399-413. doi:10.1681/ ASN.2014070673

72. Kusunoki Y, Nakazawa D, Shida H, Hattanda F, Miyoshi A, Masuda S, et al. Peptidylarginine deiminase inhibitor suppresses neutrophil extracellular trap formation and MPO-ANCA production. Front Immunol (2016) 7:227. doi:10.3389/fimmu.2016.00227

73. Bawadekar M, Shim D, Johnson CJ, Warner TF, Rebernick R, Damgaard D, et al. Peptidylarginine deiminase 2 is required for tumor necrosis factor alpha-induced citrullination and arthritis, but not neutrophil extracellular trap formation. J Autoimmun (2017) 80:39-47. doi:10.1016/j.jaut.2017.01.006

74. Obermayer A, Stoiber W, Krautgartner WD, Klappacher M, Kofler B, Steinbacher $\mathrm{P}$, et al. New aspects on the structure of neutrophil extracellular traps from chronic obstructive pulmonary disease and in vitro generation. PLoS One (2014) 9:e97784. doi:10.1371/journal.pone.0097784

75. Kolaczkowska E, Jenne CN, Surewaard BG, Thanabalasuriar A, Lee WY, Sanz MJ, et al. Molecular mechanisms of NET formation and degradation revealed by intravital imaging in the liver vasculature. Nat Commun (2015) 6:6673. doi:10.1038/ncomms7673

76. Pilsczek FH, Salina D, Poon KK, Fahey C, Yipp BG, Sibley CD, et al. A novel mechanism of rapid nuclear neutrophil extracellular trap formation in response to Staphylococcus aureus. J Immunol (2010) 185:7413-25. doi:10.4049/ jimmunol.1000675

77. Desai J, Mulay SR, Nakazawa D, Anders HJ. Matters of life and death. How neutrophils die or survive along NET release and is "NETosis" = necroptosis? Cell Mol Life Sci (2016) 73:2211-9. doi:10.1007/s00018-016-2195-0

78. Malachowa N, Kobayashi SD, Freedman B, Dorward DW, DeLeo FR. Staphylococcus aureus leukotoxin GH promotes formation of neutrophil extracellular traps. J Immunol (2013) 191:6022-9. doi:10.4049/jimmunol.1301821

79. Gonzalez DJ, Corriden R, Akong-Moore K, Olson J, Dorrestein PC, Nizet V. $\mathrm{N}$-terminal ArgD peptides from the classical Staphylococcus aureus Agr system have cytotoxic and proinflammatory activities. Chem Biol (2014) 21:1457-62. doi:10.1016/j.chembiol.2014.09.015

80. Desai J, Foresto-Neto O, Honarpisheh M, Steiger S, Nakazawa D, Popper B, et al. Particles of different sizes and shapes induce neutrophil necroptosis 
followed by the release of neutrophil extracellular trap-like chromatin. Sci Rep (2017) 7:15003. doi:10.1038/s41598-017-15106-0

81. Zawrotniak M, Bochenska O, Karkowska-Kuleta J, Seweryn-Ozog K, Aoki W, Ueda M, et al. Aspartic proteases and major cell wall components in Candida albicans trigger the release of neutrophil extracellular traps. Front Cell Infect Microbiol (2017) 7:414. doi:10.3389/fcimb.2017.00414

82. Moyes DL, Wilson D, Richardson JP, Mogavero S, Tang SX, Wernecke J, et al. Candidalysin is a fungal peptide toxin critical for mucosal infection. Nature (2016) 532:64-8. doi:10.1038/nature17625

83. Franke A, Kasper L, Hoefs S, Mogavero S, Wilson D, Hube B. Role of the pore-forming toxin ecel in candida albicans - macrophage interaction. Mycoses (2015) 58(S3):42-3.
Conflict of Interest Statement: The authors declare that the research was conducted in the absence of any commercial or financial relationships that could be construed as a potential conflict of interest.

Copyright $\odot 2018$ Guiducci, Lemberg, Küng, Schraner, Theocharides and LeibundGutLandmann. This is an open-access article distributed under the terms of the Creative Commons Attribution License (CC BY). The use, distribution or reproduction in other forums is permitted, provided the original author $(s)$ and the copyright owner(s) are credited and that the original publication in this journal is cited, in accordance with accepted academic practice. No use, distribution or reproduction is permitted which does not comply with these terms. 